\title{
Finite-Time Stabilization for $p$-Norm Stochastic Nonlinear Systems with Output Constraints
}

\author{
Shijun Guan ${ }^{1}$ and Liandi Fang $\mathbb{D D}^{2,3}$ \\ ${ }^{1}$ Department of Economic Management, Anhui Vocational College of Press and Publishing, Hefei 230601, China \\ ${ }^{2}$ College of Mathematics and Computer Science, Tongling University, Tongling 244000, China \\ ${ }^{3}$ School of Electrical and Information Engineering, Jiangsu University, Zhenjiang 212013, China
}

Correspondence should be addressed to Liandi Fang; fangld@tlu.edu.cn

Received 3 June 2020; Revised 7 July 2020; Accepted 10 July 2020; Published 12 August 2020

Guest Editor: Xiaodi Li

Copyright (C) 2020 Shijun Guan and Liandi Fang. This is an open access article distributed under the Creative Commons Attribution License, which permits unrestricted use, distribution, and reproduction in any medium, provided the original work is properly cited.

\begin{abstract}
This paper investigates the finite-time stability problem of $p$-norm stochastic nonlinear systems subject to output constraint. To cope with the constraint on system output, a tan-type barrier Lyapunov function (BLF) is constructed. By using the constructed BLF and the backstepping technique, a new control algorithm is proposed with a continuous state-feedback controller being designed, which guarantees not only that the requirement of output constraint is always achieved but also that the origin of the system is finite-time stable. This result is demonstrated by both the rigorous analysis and the simulation example.
\end{abstract}

\section{Introduction}

During the past decades, the control problem of nonlinear systems has long been a hot topic, and many control design approaches have been proposed for various kinds of nonlinear systems, such as adaptive fuzzy control $[1,2]$, output tracking control $[3,4], H_{\infty}$ control $[5,6]$, and sliding mode control [7-10]. Due to their important roles in many science and industry applications, the stochastic nonlinear systems have attracted much interest in recent years. With the development of stochastic theory, various control design strategies have been developed for types of stochastic nonlinear systems by the backstepping technique, see [11-14], for examples. Especially, some works have considered $p$-norm stochastic nonlinear systems, which are inherently nonlinear due to the fractional powers of such systems being not identically equal to one. It should be noted that the inherent nonlinearities cause the stability and control design problems, which are not very easy to be solved [15]. Luckily, the issues have been well studied for p-norm stochastic nonlinear systems with different structures by the adding a power integrator technique in the existing literatures. For instance, $\mathrm{Li}$ et al. [16] have considered the adaptive state-feedback stabilization for $p$-norm stochastic nonlinear systems; the output-feedback control has been addressed for $p$-norm stochastic nonlinear systems with time-varying delays in [17]; Zhao et al. [18] have proposed a neural tracking control algorithm for $p$-norm switched stochastic nonlinear systems. More latest studies can be found in [19-21] and the references within.

However, most of the abovementioned works about p-norm stochastic nonlinear systems did not take the output constraint into consideration. As it is well known, many actual systems are subject to output constraint due to the consideration of the system performance and operation safety $[22,23]$. For this reason, the constrained control issue of nonlinear systems has drawn attention from many scholars. Tee et al. [24] have first proposed the notion of the barrier Lyapunov function (BLF) and consequently have developed a control design strategy for a class of strictfeedback deterministic nonlinear systems with output constraints. After then, with the aid of BLFs, control design schemes have been presented for many deterministic nonlinear systems with different types of constraints, including stability control for nonlinear systems with time-varying or asymmetric output constraints $[25,26]$, adaptive control for 
nonlinear systems with full-state constrains [27], and sliding mode control for nonlinear systems with output constraints [28-30]. Moreover, since the finite-time control possesses some inherent advantages [31-33], techniques for the finitetime stabilization under output/state constraints have also been developed, respectively, for strict-feedback nonlinear systems [34], norm nonlinear systems [35-37], and switched nonlinear systems [38]. On the basis of these results, the constrained control schemes for some classes of stochastic nonlinear systems have also been proposed. Jin [39] has constructed an adaptive tracking controller for a class of output-constrained stochastic nonlinear systems in strictfeedback form. Later, the adaptive control problem and the finite-time control problem have been, respectively, addressed for stochastic nonlinear systems with full-state constraints in [40,41]. Furthermore, the adaptive neural network or fuzzy constrained control problems have attracted some attention [42-46]. Nevertheless, the stochastic nonlinear systems with output constraints considered in most of the existing related works are in the strictfeedback form, rather than in $p$-normal form. On the contrary, the existing research has mainly focused on the adaptive control problem but did not take the finite-time stabilization into account.

Motivated by the above discussions, we will investigate the problem of the finite-time stabilization for a class of $p$-norm stochastic nonlinear systems with output constraints and unknown time-varying parameters. First of all, a BLF-based control strategy will be developed by the backstepping approach. Secondly, applying stochastic Lyapunov theorems and $I t \hat{O}$ 's formula, the constructed state-feedback controller is rigorously proved to be able to ensure the achievement of the output constraint and the finite-time stability of the considered systems simultaneously. Finally, the main result of this paper will be further demonstrated by a simulation example.

\section{Problem and Preliminaries}

2.1. Problem Statement. The following class of stochastic nonlinear systems are considered:

$$
\begin{aligned}
\mathrm{d} x_{i}= & \vartheta_{i}(t) x_{i+1}^{q_{i}} \mathrm{~d} t+f_{i}\left(\bar{x}_{i}\right) \mathrm{d} t+g_{i}^{T}\left(\bar{x}_{i}\right) \mathrm{d} \omega, \\
& i=1, \ldots, n-1, \\
\mathrm{~d} x_{n}= & \vartheta_{n}(t) u^{q_{n}} \mathrm{~d} t+f_{n}\left(\bar{x}_{n}\right) \mathrm{d} t+g_{n}^{T}\left(\bar{x}_{n}\right) \mathrm{d} \omega, \\
y= & x_{1},
\end{aligned}
$$

where $\omega$ is a $N$-dimension standard Wiener process; $\bar{x}_{i}=$ $\left(x_{1}, \ldots, x_{i}\right)^{T} \in R^{i}, u \in R$ and $y \in R$ are system state, control input, and output, respectively; $\vartheta_{i}(t)$ is the time-varying parameter; the nonlinear functions $f_{i}: R^{i} \longrightarrow R$ and $g_{i}: R^{i} \longrightarrow R^{N}$ are continuous and satisfy $f_{i}(0)=g_{i}(0)=0$; and the fractional powers $q_{i}$ 's meet the requirement $q_{i} \in R_{\text {odd }}^{\geq 1}:=\{\tau \geq 1, \tau$ is the positive odd integersratio $\}$. The output $y$ is required to satisfy

$$
y \in \prod_{1}=\{y(t) \in R,|y(t)|<b\}=\left\{x_{1} \in R,\left|x_{1}(t)\right|<b\right\},
$$

where $b$ is a known positive constant.

This paper aims to design a continuous state-feedback controller for system (1), which can ensure that the origin of the closed-loop system is finite-time stable in probability and the requirement of the output constraint is achieved.

\subsection{Preliminaries}

Notations 1. For $k=1, \ldots, n$, let $g_{k}^{-T}\left(\bar{x}_{k}\right)=\left(g_{1}^{T}\left(x_{1}\right), \ldots, g_{i}^{T}\right.$ $\left.\left(\bar{x}_{k}\right)\right)$ and $\Pi_{k}=\left\{\bar{x}_{k} \in R^{k},\left|x_{1}(t)\right|<b\right\}$. For any $\varsigma \in R$ and $\theta>0$, denote $\varphi(\varsigma)=[\varsigma]^{\theta}:=|\varsigma|^{\theta} \operatorname{sgn}(\varsigma)$.

Consider the following stochastic system:

$$
\mathrm{d} x=f(x) \mathrm{d} t+g(x) \mathrm{d} \omega,
$$

where $f(x)$ and $g(x)$ are continuous satisfying $f(0)=0$ and $g(0)=0$.

Definition 1 (see [13]). For any given $V(x) \in C^{2}\left(R^{n}\right)$, associated with system (1), the second-order differential operator is defined as follows:

$$
\ell V=\frac{\partial V}{\partial x} f(x)+\frac{1}{2} \operatorname{tr}\left\{g^{T}(x) \frac{\partial^{2} V}{\partial x^{2}} g(x)\right\} .
$$

Definition 2 (see [24]). Suppose that $\Pi$ is an open set containing the origin and $V: \Pi \longrightarrow R$ is positive definite and continuously differentiable. Then, for system $\dot{x}=$ $g(x), V(x(t))$ is called a BLF if for each solution $x(t)$ starting from $x\left(t_{0}\right) \in \Pi, V(x(t)) \longrightarrow \infty$, as $x(t) \longrightarrow \partial \Pi$, $V(x(t)) \leq \tau$ for all $t \geq t_{0}$ and for some $\tau \in R^{+}$.

Assumption 1 (see [36]). For $\forall i=1, \ldots, n$, there exist known positive constants $\underline{\vartheta}_{i}$ and $\bar{\vartheta}_{i}$ such that $\underline{\vartheta}_{i} \leq \mathcal{\vartheta}_{i}(t) \leq \bar{\vartheta}_{i}$.

Assumption 2. For $i=1, \ldots, n$, there are a constant $\mu \in\left(-\left[1+\sum_{j=2}^{n} q_{1} \cdots q_{j-1}\right]^{-1}, 0\right)$ and known nonnegative smooth functions $\psi_{i}\left(\bar{x}_{i}\right), \eta_{i}\left(\bar{x}_{i}\right)$ such that

$$
\begin{aligned}
& \left|f_{i}\left(\bar{x}_{i}\right)\right| \leq \psi_{i}\left(\bar{x}_{i}\right) \sum_{j=1}^{i}\left|x_{j}\right|^{\left(v_{i}+\mu / v_{j}\right)}, \\
& \left\|g_{i}\left(\bar{x}_{i}\right)\right\| \leq \eta_{i}\left(\bar{x}_{i}\right) \sum_{j=1}^{i}\left|x_{j}\right|^{\left(2 v_{i}+\mu / 2 v_{j}\right)},
\end{aligned}
$$

for all $t \geq 0$, where $v_{1}=1, v_{j+1}=\left(\left(v_{j}+\mu\right) / q_{j}\right)>0, j=1, \ldots$, $n$.

Remark 1. Note that condition (4) is borrowed from [34]. However, the systems considered in [34] are p-norm deterministic nonlinear systems, while we consider $p$-norm stochastic nonlinear systems with drift terms $f_{i}$ 's and 
diffusion terms $g_{i}$ 's in this paper. In light of $\mu \in\left(-\left[1+\sum_{j=2}^{n} q_{1} \cdots q_{j-1}\right]^{-1}, 0\right)$, the value of $\mu$ is generally taken as $\mu=-(m / p)$ for simplicity, where $m$ and $p$ represent even and odd integers, respectively. Then, the value of each $v_{j}(j=2, \ldots, n)$ can be obtained by applying $v_{1}=1$ and $v_{j+1}=\left(\left(v_{j}+\mu\right) / q_{j}\right)>0$. It can also be observed that both the denominator and numerator of each $v_{j}$ are odd.

Lemma 1 (see [13]). Suppose that there exists a positive Lyapunov function $V \in C^{2}\left(R^{n}\right)$, which satisfies $\lim _{|x| \longrightarrow \infty}$ $V(x)=\infty$. If $\ell V$ is with respect to (3) and satisfies $\ell V \leq 0, \forall x \in R^{n}$, then system (3) has a solution for any initial value.

Lemma 2 (see [13]). Suppose that system (3) admits a solution for each initial value. If there are $\kappa_{\infty}$ class functions $\varrho_{1}(\cdot)$ and $\varrho_{2}(\cdot)$, a positive $C^{2}$ Lyapunov function $V$, real numbers $c>0$, and $0<\gamma<1$, such that

$$
\begin{gathered}
\varrho_{1}(|x|) \leq V(x) \leq \varrho_{2}(|x|), x \in R^{n}, \\
\ell V(x) \leq-c V^{\gamma}(x), x \in R^{n} \backslash\{0\} .
\end{gathered}
$$

For all $t \geq 0$, then the origin of system (3) is finite-time stable in probability.

Lemma 3 (see [37]). Let $a, b, \rho$, and $\varsigma$ be positive real numbers. For any $z_{1}, z_{2} \in R$, we have

$$
\rho\left|z_{1}\right|^{a}\left|z_{2}\right|^{b} \leq \varsigma \frac{a}{a+b}\left|z_{1}\right|^{a+b}+\frac{b}{a+b} \rho^{a+b / b} \varsigma^{-a / b}\left|z_{2}\right|^{a+b} .
$$

Lemma 4 (see [8]). Let $p \in(0, \infty)$; for any $\varsigma_{i} \in R, i=1, \ldots$, $n$, one has

$$
\left(\left|\varsigma_{1}\right|+\cdots+\left|\varsigma_{n}\right|\right)^{p} \leq d\left(\left|\varsigma_{1}\right|^{p}+\cdots+\left|\varsigma_{n}\right|^{p}\right),
$$

where $d=n^{p-1}$ if $p \geq 1$ and $d=1$ if $0<p<1$.

Lemma 5 (see [10]). Leta, $d \in R^{+}$with $a \geq 1$. For any $\varsigma_{1}, \varsigma_{2} \in R$, we have
(i) $\left|\varsigma_{1}^{a}-\varsigma_{2}^{a}\right| \leq a\left(2^{a-2}+2\right)\left|\varsigma_{1}-\varsigma_{2}\right|\left(\left|\varsigma_{1}-\varsigma_{2}\right|^{a-1}+\varsigma_{2}^{a-1}\right)$
(ii) $\left.\left|\varsigma_{1}^{(d / a)}-\varsigma_{2}^{(d / a)}\right| \leq 2^{1-(1 / a)}|| \varsigma_{1}\right]^{d}-\left\lceil\left.\left.\varsigma_{2}\right|^{d}\right|^{(1 / a)}\right.$
(iii) $\left(\left|\varsigma_{1}\right|+\left|\varsigma_{2}\right|\right)^{1 / a} \leq\left|\varsigma_{1}\right|^{1 / a}+\left|\varsigma_{2}\right|^{1 / a} \leq 2^{1-(1 / a)}\left(\left|\varsigma_{1}\right|+\right.$ $\left.\left|\varsigma_{2}\right|\right)^{1 / a}$

\section{Main Results}

3.1. A Tan-Type BLF. Before carrying out the control design for system (1), we should handle the output constraint issue.

Firstly, we denote $v_{0}=\max _{1 \leq i \leq n}\left\{v_{i}\right\}$ and $c_{i}=\left(v_{0} / v_{i}\right)(i=1, \ldots, n)$. Let $\sigma$ be a constant parameter satisfying $\sigma \geq \sigma_{0}$, where the value of $\sigma_{0}$ is chosen as below:

(i) If for all $2 \leq i \leq n, 1 \leq c_{i} \leq 2$, then $\sigma_{0}=2 v_{0}$

(ii) If for all $2 \leq i \leq n, c_{i} \geq 2$, then $\sigma_{0}=v_{0}$

Consequently, it is clear that $\left(\sigma / v_{i}\right) \geq 2$.

Then, a tan-type BLF can be constructed on $\Pi_{1}$ as follows:

$$
V_{b}\left(x_{1}\right)=\frac{2 b^{4 \sigma-\mu}}{(4 \sigma-\mu) \pi} \tan \left(\frac{\pi\left|x_{1}\right|^{4 \sigma-\mu}}{2 b^{4 \sigma-\mu}}\right)
$$

where $\mu$ is given by Assumption 2 and $\sigma$ is defined as above.

It is not hard to obtain from the expression of $V_{b}\left(x_{1}\right)$ that

$$
\begin{aligned}
\frac{\partial V_{b}}{\partial x_{1}}= & \sec ^{2}\left(\frac{\pi\left|x_{1}\right|^{4 \sigma-\mu}}{2 b^{4 \sigma-\mu}}\right)\left\lceil x_{1}\right\rceil^{4 \sigma-\mu-1}=G\left(x_{1}\right)\left\lceil x_{1}\right\rceil^{4 \sigma-\mu-1}, \\
\frac{\partial^{2} V_{b}}{\partial x_{1}^{2}}= & (4 \sigma-\mu-1) G\left(x_{1}\right)\left|x_{1}\right|^{4 \sigma-\mu-2} \\
& +\frac{\pi(4 \sigma-\mu)}{b^{4 \sigma-\mu}} G\left(x_{1}\right) \tan \left(\frac{\pi\left|x_{1}\right|^{4 \sigma-\mu}}{2 b^{4 \sigma-\mu}}\right)\left|x_{1}\right|^{2(4 \sigma-\mu-1)},
\end{aligned}
$$

where $G\left(x_{1}\right)=\sec ^{2}\left(\left(\pi\left|x_{1}\right|^{4 \sigma-\mu}\right) / 2 b^{4 \sigma-\mu}\right)$.

Remark 2. It should be noted that the BLF is modified from [34], which is constructed by fully taking the advantage of the given nonlinear growth conditions. As stated in [34], the control strategy based on $V_{b}\left(x_{1}\right)$ is a universal method, which can handle stochastic systems with or without output constraints.

3.2. Controller Design and Stability Analysis. In what follows, a continuous state-feedback controller will be constructed, and the stability of system (1) under the designed controller will be rigorously analysed. To this end, a theorem is presented to describe the main result.

Theorem 1. Suppose Assumptions 1-2 hold for system (1). For any constant $b>0$, there is a continuous state-feedback controller such that

(i) The output of system (1) is kept in a given constrained set in the sense of probability, i.e., $P\{|y(t)|<b\}=1$

(ii) The origin of the closed-loop system is finite-time stable in probability.

Proof. The proof contains three parts. First of all, the design procedure of the controller is explicitly displayed. Then, the system output is proved to be kept in the given constrained set with probability one. In the last part, the finite-time stability of system (1) is rigorously analysed.

\subsubsection{Part I: Design Procedure}

Step 1. Let $\varsigma_{1}=\left\lceil x_{1}\right\rceil^{\sigma}$, and choose the Lyapunov function $V_{1}\left(x_{1}\right)=V_{b}\left(x_{1}\right)$. Then, we can directly get from Definition 1 that 


$$
\begin{aligned}
\ell V_{1} \leq & \vartheta_{1}(t) G\left(x_{1}\right)\left\lceil x_{1}\right\rceil^{4 \sigma-\mu-1} x_{2}^{q_{1}}+\frac{4 \sigma-\mu-1}{2} \\
& \cdot G\left(x_{1}\right)\left|x_{1}\right|^{4 \sigma-\mu-2} \eta_{1}^{2}\left|x_{1}\right|^{2+\mu} \\
+ & \bar{\vartheta}_{1} G\left(x_{1}\right)\left|x_{1}\right|^{4 \sigma-\mu-1} \psi_{1}\left|x_{1}\right|^{1+\mu}+\frac{\pi(4 \sigma-\mu)}{2 b^{4 \sigma-\mu}} \\
& \cdot\left|x_{1}\right|^{2(4 \sigma-\mu-1)} G\left(x_{1}\right) \tan \left(\frac{\pi\left|x_{1}\right|^{4 \sigma-\mu}}{2 b^{4 \sigma-\mu}}\right) \eta^{2}\left|x_{1}\right|^{2+\mu} \\
\leq & \vartheta_{1}(t) G\left(x_{1}\right)\left\lceil\varsigma_{1}\right\rceil^{((4 \sigma-\mu-1) / \sigma)} \xi_{2}^{q_{1}}+\vartheta_{1}(t) \\
& \cdot G\left(x_{1}\right)\left\lceil\varsigma_{1}\right\rceil^{((4 \sigma-\mu-1) / \sigma)}\left(x_{2}^{q_{1}}-\xi_{2}^{q_{1}}\right)+H_{1}\left(x_{1}\right) G\left(x_{1}\right) \varsigma_{1}^{4},
\end{aligned}
$$

where $\quad H_{1}\left(x_{1}\right) \geq \bar{\vartheta}_{1} \psi_{1}+(1 / 2) \eta_{1}^{2}(4 \sigma-\mu-1)+(\pi(4 \sigma-\mu)$ $\left.\eta_{1}^{2} / 2 b^{4 \sigma-\mu}\right) \tan \left(\pi\left|x_{1}\right|^{4 \sigma-\mu} / 2 b^{4 \sigma-\mu}\right)\left|x_{1}\right|^{4 \sigma-\mu}$ is a nonnegative $C^{2}$ function and $\xi_{2}$ is a virtual controller required to be design after later.

Then, we design

$\xi_{2}=-\lambda_{1}\left(x_{1}\right)\left\lceil\varsigma_{1}\right\rceil^{\left(v_{2} / \sigma\right)}$ with $\lambda_{1}\left(x_{1}\right)=\left(\frac{n+H_{1}\left(x_{1}\right)}{\underline{\vartheta}_{1}}\right)^{\left(1 / q_{1}\right)}>0$.
Substituting (12) into (13) yields

$$
\begin{aligned}
\ell V_{1}\left(x_{1}\right) \leq & -n G\left(x_{1}\right) \varsigma_{1}^{4}+\vartheta_{1}(t) G\left(x_{1}\right)\left\lceil\varsigma_{1}\right\rceil^{((4 \sigma-\mu-1) / \sigma)} \\
& \cdot\left(x_{2}^{q_{1}}-\xi_{2}^{q_{1}}\right) \\
= & -\frac{1}{2} G\left(x_{1}\right) \varsigma_{1}^{4}-\left(n-\frac{1}{2}\right) G\left(x_{1}\right) \varsigma_{1}^{4}+\vartheta_{1}(t) \\
& \cdot G\left(x_{1}\right)\left\lceil\varsigma_{1}\right\rceil^{((4 \sigma-\mu-1) / \sigma)}\left(x_{2}^{q_{1}}-\xi_{2}^{q_{1}}\right) \\
\leq & -\frac{1}{2} G\left(x_{1}\right) \varsigma_{1}^{4}-\left(n-\frac{1}{2}\right) \varsigma_{1}^{4}+\vartheta_{1}(t) \\
& \cdot G\left(x_{1}\right)\left\lceil\varsigma_{1}\right\rceil^{((4 \sigma-\mu-1) / \sigma)}\left(x_{2}^{q_{1}}-\xi_{2}^{q_{1}}\right) .
\end{aligned}
$$

Step 2. We denote $\varsigma_{2}=\left\lceil x_{2}\right\rceil^{\sigma / v_{2}}-\left\lceil\xi_{2}\right\rceil^{\sigma / v_{2}}$ and define the positive Lyapunov function $V_{2}$ on $\Pi_{2}$ as $V_{2}=V_{1}+\Psi_{2}$ with

$$
\Psi_{2}=\int_{\xi_{2}}^{x_{2}}\left\lceil\lceil r\rceil^{\left(\sigma / v_{2}\right)}-\left\lceil\xi_{2}\right\rceil^{\left(\sigma / v_{2}\right)}\right\rceil^{((4 \sigma-\mu-1) / \sigma)} \mathrm{d} r .
$$

Since $\left(\partial\left\lceil\xi_{2}\right\rceil^{\left(\sigma / v_{2}\right)} / \partial x_{1}\right)=-\left(\partial \lambda_{1}^{\left(\sigma / v_{2}\right)}\left(x_{1}\right) /\left\lceil x_{1}\right\rceil^{\sigma}-\sigma \lambda_{1}^{\left(\sigma / v_{2}\right)}\right.$ $\left(x_{1}\right)\left|x_{1}\right|^{(\sigma-1)}$ is valid, one can obtain

$$
\begin{aligned}
\frac{\partial \Psi_{2}}{\partial x_{2}}= & \left\lceil\varsigma_{2}\right\rceil^{((4 \sigma-\mu-1) / \sigma)}, \\
\frac{\partial \Psi_{2}}{\partial x_{1}}= & \frac{-4 \sigma-\mu-v_{2}}{\sigma} \frac{\partial\left\lceil\xi_{2}\right\rceil^{\left(\sigma / v_{2}\right)}}{\partial x_{1}} \int_{\xi_{2}}^{x_{2}}\left|\lceil r\rceil^{\left(\sigma / v_{2}\right)}-\left\lceil\xi_{2}\right\rceil^{\left(\sigma / v_{2}\right)}\right|^{\left(\left(3 \sigma-\mu-v_{2}\right) / \sigma\right)} \mathrm{d} r, \\
\frac{\partial^{2} \Psi_{2}}{\partial x_{1} \partial x_{2}}= & \frac{4 \sigma-\mu-v_{2}}{\sigma}\left\lceil\varsigma_{2}\right\rceil^{\left(\left(3 \sigma-\mu-v_{2}\right) / \sigma\right.} \frac{\partial\left\lceil\xi_{2}\right\rceil^{\left(\sigma / v_{2}\right)}}{\partial x_{1}}, \\
\frac{\partial^{2} \Psi_{2}}{\partial x_{2}^{2}}= & \frac{4 \sigma-\mu-v_{2}}{v_{2}}\left|x_{2}\right|^{\left(\sigma-v_{2}\right) / v_{2}}\left\lceil\varsigma_{2}\right\rceil^{\left(\left(3 \sigma-\mu-v_{2}\right) / \sigma\right)}, \\
\frac{\partial^{2} \Psi_{2}=}{\partial x_{1}^{2}=} & \frac{-4 \sigma-\mu-v_{2}}{\sigma} \frac{\partial^{2}\left\lceil\xi_{2}\right\rceil^{\left(\sigma / v_{2}\right)}}{\partial x_{1}^{2}} \int_{\xi_{2}}^{x_{2}}\left|\lceil r\rceil^{\left(\sigma / v_{2}\right)}-\left\lceil\xi_{2}\right\rceil^{\sigma / v_{2}}\right|^{\left(\left(3 \sigma-\mu-v_{2}\right) / \sigma\right)} \mathrm{d} r \\
& +\frac{4 \sigma-\mu-v_{2}}{\sigma} \times \frac{3 \sigma-\mu-v_{2}}{\sigma}\left(\frac{\partial\left\lceil\left.\xi_{2}\right|^{\sigma / v_{2}}\right.}{\partial x_{1}}\right)^{2} \int_{\xi_{2}}^{x_{2}}\left|\lceil r\rceil^{\left(\sigma / v_{2}\right)}-\left\lceil\xi_{2}\right\rceil^{\left(\sigma / v_{2}\right)}\right|^{\left(\left(2 \sigma-\mu-v_{2}\right) / \sigma\right)} \mathrm{d} r .
\end{aligned}
$$

Using Definition 1 again, we have

$$
\begin{aligned}
\ell V_{2} \leq & -\frac{1}{2} G\left(x_{1}\right) \zeta_{1}^{4}-\left(n-\frac{1}{2}\right) \zeta_{1}^{4}+\vartheta_{1}(t) G\left(x_{1}\right)\left[x_{1}\right]^{4 \sigma-\mu-1}\left(x_{2}^{q_{1}}-\xi_{2}^{q_{1}}\right)+\frac{\partial \Psi_{2}}{\partial x_{1}}\left(\vartheta_{1}(t) x_{2}^{q_{1}}+f_{1}\left(x_{1}\right)\right) \\
& +\frac{\partial \Psi_{2}}{\partial x_{1}} f_{2}\left(\bar{x}_{2}\right)+\frac{1}{2} \operatorname{tr}\left\{\bar{g}_{2}^{T} \frac{\partial^{2} \Psi_{2}}{\partial \bar{x}_{2}^{2}} \bar{g}_{2}\right\}+\frac{\partial \Psi_{2}}{\partial x_{2}} \vartheta_{2}(t) \xi_{3}^{q_{2}}+\frac{\partial \Psi_{2}}{\partial x_{2}} \vartheta_{2}(t)\left(x_{3}^{q_{2}}-\xi_{3}^{q_{2}}\right),
\end{aligned}
$$


where $\xi_{3}$ is the virtual controller required to be designed later.

In the following, each term in the right hand of (16) will be estimated by its upper bound.

Firstly, applying $0<\left(v_{2} / \sigma\right) \leq 1,0<((1+\mu) / \sigma) \leq 1$ and Lemma 5 , it is easily obtained that

$$
\left|x_{2}-\xi_{2}\right| \leq \mid\left\lceil\left. x_{2}\right|^{\sigma / v_{2}}-\left\lceil\left.\left.\xi_{2}\right|^{\sigma / v_{2}}\right|^{v_{2} / \sigma} \times 2^{1-\left(v_{2} / \sigma\right)} \leq 2\left|\zeta_{2}\right|^{v_{2} / \sigma},\right.\right.
$$

$$
\left|x_{2}\right|^{q_{1}} \leq\left|\zeta_{2}+\left\lceil\xi_{2}\right]^{\sigma / v_{2}}\right|^{(1+\mu) / \sigma} \leq\left|\zeta_{2}\right|^{(1+\mu) / \sigma}+\lambda_{1}{ }^{(1+\mu) / v_{2}}\left|\zeta_{2}\right|^{(1+\mu) / \sigma},
$$

$$
\left|x_{2}^{q_{1}}-\xi_{2}^{q_{1}}\right| \leq 2^{1-(1+\mu) / \sigma}\left|\left\lceil x_{2}\right]^{\sigma / v_{2}}-\left\lceil\xi_{2}\right]^{\sigma / v_{2}}\right|^{(1+\mu) / \sigma} \leq 2\left|\zeta_{2}\right|^{(1+\mu) / \sigma} .
$$

It can be deduced from (19) and Lemma 3 that

$$
\begin{aligned}
& \vartheta_{1}(t) G\left(x_{1}\right)\left[x_{1}\right]^{4 \sigma-\mu-1}\left(x_{2}^{q_{1}}-\xi_{2}^{q_{1}}\right) \leq 2 \bar{\vartheta}_{1} G\left(x_{1}\right)\left|\zeta_{1}\right|^{(4 \sigma-\mu-1) / \sigma} \\
& \cdot\left|\zeta_{2}\right|^{(\mu+1) / \sigma} \leq \frac{1}{8} \zeta_{1}^{4}+H_{21}\left(\bar{x}_{2}\right) \zeta_{2}^{4},
\end{aligned}
$$

where $\quad H_{21}\left(\bar{x}_{2}\right) \geq((\mu+1) / 4 \sigma)\left(2 \bar{\vartheta}_{1} G\left(x_{1}\right)\right)^{4 \sigma /(\mu+1)}(4 \sigma /$ $(8(4 \sigma-\mu-1)))^{-(4 \sigma-\mu-1) / 1+\mu} \geq 0$ is a $C^{2}$ function.

Secondly, one can obtain from Assumption 1 and Lemma 4 that

$$
\begin{aligned}
\left|f_{2}\left(\bar{x}_{2}\right)\right| \leq & \widetilde{\psi}_{2}\left(\bar{x}_{2}\right)\left[\left|x_{1}\right|^{\left(v_{2}+\mu\right) / v_{1}}+\left|x_{2}\right|^{\left(v_{2}+\mu\right) / v_{2}}\right] \\
& \leq \widetilde{\psi}_{2}\left(\bar{x}_{2}\right)\left[\left|\zeta_{1}\right|^{\left(v_{2}+\mu\right) / \sigma}+\lambda_{1}^{\left(v_{2}+\mu\right) / v_{2}}\left|\zeta_{1}\right|^{\left(v_{2}+\mu\right) / \sigma}\right. \\
& \left.+\left|\zeta_{2}\right|^{\left(v_{2}+\mu\right) / \sigma}\right] \\
\leq & \widetilde{\psi}_{2}\left(\bar{x}_{2}\right)\left[\left|\zeta_{1}\right|^{\left(v_{2}+\mu\right) / \sigma}+\zeta_{2}^{\left(v_{2}+\mu\right) / \sigma}\right] \\
\left\|g_{2}\left(\bar{x}_{2}\right)\right\| \leq & \eta_{2}\left(\bar{x}_{2}\right)\left[\left|x_{1}\right|^{\left(2 v_{2}+\mu\right) / 2 v_{1}}+\left|x_{2}\right|^{\left(2 v_{2}+\mu\right) / 2 v_{2}}\right] \\
\leq & \eta_{2}\left(\bar{x}_{2}\right)\left[\left|\zeta_{1}\right|^{\left(2 v_{2}+\mu\right) / 2 \sigma}+\lambda_{1}^{\left(2 v_{2}+\mu\right) / 2 v_{2}}\left|\zeta_{1}\right|^{\left(2 v_{2}+\mu\right) / 2 \sigma}\right. \\
& \left.+\left|\zeta_{2}\right|^{\left(2 v_{2}+\mu\right) / 2 \sigma}\right] \leq \widetilde{\eta}_{2}\left(\bar{x}_{2}\right) \\
& \cdot\left[\left|\zeta_{1}\right|^{\left(2 v_{2}+\mu\right) / 2 \sigma}+\left|\zeta_{2}\right|^{\left(2 v_{2}+\mu\right) / 2 \sigma}\right]
\end{aligned}
$$

where $\widetilde{\psi}_{2}\left(\bar{x}_{2}\right) \geq \psi_{2}\left(\bar{x}_{2}\right)\left(1+\lambda_{1}^{\left(v_{2}+\mu\right) / v_{2}}\right)$ and $\widetilde{\eta}_{2}\left(\bar{x}_{2}\right) \geq$ $\eta_{2}\left(\bar{x}_{2}\right)\left(1+\lambda_{1}^{\left(2 v_{2}+\mu\right) / 2 v_{2}}\right)$ are nonnegative smooth functions.
Additionally, note that $\left(\sigma / v_{2}\right) \geq 2$. Then, as stated in [36], there exist $C^{2}$ functions $M_{21}\left(\bar{x}_{2}\right) \geq 0, K_{21}\left(\bar{x}_{2}\right) \geq 0$, and $\widetilde{M}_{21}\left(\bar{x}_{2}\right) \geq 0$, such that

$$
\left|\frac{\partial\left\lceil\xi_{2}\right]^{\sigma / v_{2}}}{\partial x_{1}^{2}}\right| \leq\left|\frac{\partial \lambda_{1}^{\sigma / v_{2}}}{\partial x_{1}}\right|\left|x_{1}\right|^{\sigma}+\sigma \lambda_{1}^{\sigma / v_{2}}\left|x_{1}\right|^{\sigma-1} \leq M_{21}\left(\bar{x}_{2}\right)\left|\zeta_{1}\right|^{1-(1 / \sigma)},
$$

$$
\begin{aligned}
\left|\frac{\partial^{2}\left\lceil\xi_{2}\right\rceil^{\sigma / v_{2}}}{\partial x_{1}^{2}}\right| \leq & \left|\frac{\partial^{2} \lambda_{1}^{\sigma / v_{2}}}{\partial x_{1}^{2}}\right|\left|x_{1}\right|^{\sigma}+2 \sigma\left|\frac{\partial \lambda_{1}^{\sigma / v_{2}}}{\partial x_{1}}\right|\left|x_{1}\right|^{\sigma-1} \\
& +\sigma(\sigma-1) \lambda_{1}^{\sigma / v_{2}}\left|x_{1}\right|^{\sigma-2} \leq \mathrm{K}_{21}\left(\bar{x}_{2}\right)\left|\zeta_{1}\right|^{1-(2 / \sigma)}
\end{aligned}
$$

$$
\begin{aligned}
\frac{\partial \Psi_{2}}{\partial x_{1}} \leq & \frac{4 \sigma-\mu-v_{2}}{\sigma}\left|\frac{\partial\left[\xi_{2}\right]^{\sigma / v_{2}}}{\partial x_{1}}\right|\left|\left\lceil x_{2}\right\rceil^{\sigma / v_{2}}-\left\lceil\xi_{2}\right]^{\sigma / v_{2}}\right|^{\left(3 \sigma-\mu-v_{2}\right) / \sigma} \\
& \cdot\left|x_{2}-\xi_{2}\right| \leq \frac{4 \sigma-\mu-v_{2}}{\sigma} \\
& \times M_{21}\left(\bar{x}_{2}\right)\left|\zeta_{1}\right|^{1-(1 / \sigma)}\left|\zeta_{2}\right|^{\left(3 \sigma-\mu-v_{2}\right) / \sigma} \times 2\left|\zeta_{2}\right|^{v_{2} / \sigma} \\
\leq & \widetilde{M}_{21}\left(\bar{x}_{2}\right)\left|\zeta_{1}\right|^{1-(1 / \sigma)}\left|\zeta_{2}\right|^{(3 \sigma-\mu) / \sigma},
\end{aligned}
$$

$$
\begin{aligned}
\frac{1}{2} \frac{\partial^{2} \Psi_{2}}{\partial x_{1}^{2} \leq} \leq & \left.\frac{4 \sigma-\mu-v_{2}}{2 \sigma}\left|\frac{\partial_{2}\left\lceil\xi_{2}\right\rceil^{\sigma / v_{2}}}{\partial x_{1}^{2}}\right| \mid x_{2}\right\rceil^{\sigma / v_{2}}-\left.\left\lceil\xi_{2}\right\rceil^{\sigma / v_{2}}\right|^{\left(3 \sigma-\mu-v_{2}\right) / \sigma} \\
& \cdot\left|x_{2}-\xi_{2}\right|+\frac{\left(4 \sigma-\mu-v_{2}\right)\left(3 \sigma-\mu-v_{2}\right)}{2 \sigma^{2}}\left(\frac{\partial\left\lceil\xi_{2}\right\rceil^{\sigma / v_{2}}}{\partial x_{1}}\right) \\
& \cdot\left|\left\lceil x_{2}\right\rceil^{\sigma / v_{2}}-\left\lceil\xi_{2}\right\rceil^{\sigma / v_{2}}\right|^{\left(2 \sigma-\mu-v_{2}\right) / \sigma}\left|x_{2}-\xi_{2}\right| \\
\leq & \frac{4 \sigma-\mu-v_{2}}{\sigma} K_{21}\left(\bar{x}_{2}\right)\left|\zeta_{1}\right|^{1-(2 / \sigma)}\left|\zeta_{2}\right|^{(3 \sigma-\mu) / \sigma} \\
& +\frac{\left(4 \sigma-\mu-v_{2}\right)\left(3 \sigma-\mu-v_{2}\right)}{\sigma^{2}} \\
& \cdot M_{21}^{2}\left(\bar{x}_{2}\right)\left|\zeta_{1}\right|^{2-(2 / \sigma)}\left|\zeta_{2}\right|^{2 \sigma-\mu / \sigma} .
\end{aligned}
$$

Then, using (18), (21), (25), Assumptions 1-2, and Lemma 3, we can infer

$$
\begin{aligned}
& \frac{\partial \Psi_{2}}{\partial x_{1}}\left(\vartheta_{1}(t) x_{2}^{q_{1}}+f_{1}\left(x_{1}\right)\right) \leq \tilde{M}_{21}\left(\bar{x}_{2}\right) \bar{\vartheta}_{1}\left|\zeta_{1}\right|^{1-(1 / \sigma)}\left|\zeta_{2}\right|^{(3 \sigma-\mu) / \sigma} \\
& \cdot\left(\left|\zeta_{2}\right|^{(1-\mu) / \sigma}+\left|\lambda_{1}\right|^{(1+\mu) / \sigma}\right) \\
&+\tilde{M}_{21}\left(\bar{x}_{2}\right)\left|\zeta_{1}\right|^{1-(1 / \sigma)}\left|\zeta_{2}\right|^{(3 \sigma-\mu) / \sigma} \\
& \cdot \widetilde{\psi}_{1}\left|\zeta_{1}\right|^{1-(1 / \sigma)} \leq \frac{1}{8} \zeta_{1}^{4}+\mathrm{H}_{22}\left(\bar{x}_{2}\right) \zeta_{2}^{4},
\end{aligned}
$$


where

$$
\begin{aligned}
H_{22}\left(\bar{x}_{2}\right) \geq & \frac{3 \sigma+1}{4 \sigma}\left(\bar{\vartheta}_{1} \widetilde{M}_{21}\right)^{(4 \sigma /(3 \sigma+1))}\left(\frac{4 \sigma}{32(\sigma-1)}\right)^{(-(\sigma-1) / 3 \sigma+1)} \\
& +\frac{3 \sigma-\mu}{4 \sigma}\left(\bar{\vartheta}_{1} \tilde{M}_{21} \lambda_{1}^{(1+\sigma) / v_{2}}\right)^{(4 \sigma /(3 \sigma-\mu))} \\
& \cdot\left(\frac{4 \sigma}{32(\sigma+\mu)}\right)^{((-(\sigma+\mu)) / 3 \sigma-\mu)} \\
& +\frac{3 \sigma-\mu}{4 \sigma}\left(\psi_{1} \tilde{M}_{21}\right)^{(4 \sigma /(3 \sigma-\mu))} \\
& \cdot\left(\frac{4 \sigma}{16(\sigma+\mu)}\right)^{((-(\sigma+\mu)) / 3 \sigma-\mu)}
\end{aligned}
$$

is a nonnegative $C^{2}$ function.

Moreover, from (19), Assumption 2, and Lemma 3, it can be deduced that

$$
\begin{aligned}
\frac{\partial \Psi_{2}}{\partial x_{2}} f_{2}\left(\bar{x}_{2}\right) & \leq\left|\zeta_{2}\right|^{\left(4 \sigma-\mu-v_{2}\right) / \sigma}\left[\widetilde{\psi}_{2}\left(\left|\zeta_{1}\right|^{\left(v_{2}+\mu\right) / \sigma}+\zeta_{2}{ }^{\left(v_{2}+\mu\right) / \sigma}\right)\right] \\
& \leq \frac{1}{8} \zeta_{1}^{4}+H_{23}\left(\bar{x}_{2}\right) \zeta_{2}^{4},
\end{aligned}
$$

where $\quad H_{23}\left(\bar{x}_{2}\right) \geq \widetilde{\psi}_{2}+\left(\left(4 \sigma-\mu-v_{2}\right) / 4 \sigma\right)\left(\widetilde{\psi}_{2}\right)^{4 \sigma /\left(4 \sigma-\mu-v_{2}\right)}$ $\left(4 \sigma /\left(8\left(\mu+v_{2}\right)\right)\right)^{\left(-\left(\mu+v_{2}\right)\right) /\left(4 \sigma-\mu-v_{2}\right)} \geq 0$ is a $C^{2}$ function.

On the contrary, it is noted that

$$
\begin{aligned}
\frac{1}{2} \operatorname{tr}\left\{\bar{g}_{2} \frac{\partial^{2} \Psi_{2}}{\partial \bar{x}_{2}^{2}} \bar{g}_{2}\right\}= & \frac{1}{2} \frac{\partial^{2} \Psi_{2}}{\partial x_{1}^{2}}\left\|g_{1}\right\|^{2}+g_{1}^{T} \frac{\partial^{2} \Psi_{2}}{\partial x_{1} \partial x_{2}} g_{2} \\
& +\frac{1}{2} \frac{\partial^{2} \Psi_{2}}{\partial x_{2}^{2}}\left\|g_{2}\right\|^{2} .
\end{aligned}
$$

Then, applying (22), (26), Assumption 2, and Lemma 3, there clearly exist nonnegative $C^{2}$ functions $H_{241}\left(\bar{x}_{2}\right), H_{242}\left(\bar{x}_{2}\right)$, and $H_{243}\left(\bar{x}_{2}\right)$ such that

$$
\begin{aligned}
\frac{1}{2} \frac{\partial^{2} \Psi_{2}}{\partial x_{1}^{2}}\left\|g_{1}\right\|^{2} \leq & \frac{4 \sigma-\mu-v_{2}}{\sigma} K_{21}\left|\zeta_{1}\right|^{1-(2 / \sigma)}\left|\zeta_{2}\right|^{(3 \sigma-\mu) / \sigma} \eta_{1}^{2}\left|\zeta_{1}\right|^{(2+\mu) / \sigma} \\
& +\frac{\left(4 \sigma-\mu-v_{2}\right)\left(3 \sigma-\mu-v_{2}\right)}{2 \sigma^{2}} \\
& \cdot M_{21}^{2}\left|\zeta_{1}\right|^{2-(2 / \sigma)}\left|\zeta_{2}\right|^{(2 \sigma-\mu) / \sigma} \eta_{1}^{2}\left|\zeta_{1}\right|^{(2+\mu) / \sigma} \\
\leq & \frac{1}{24} \zeta_{1}^{4}+H_{241}\left(\bar{x}_{2}\right) \zeta_{2}^{4},
\end{aligned}
$$

$$
\begin{aligned}
g_{1}^{T} \frac{\partial^{2} \psi_{2}}{\partial x_{1} \partial x_{2}} g_{2} \leq & \eta_{1}\left|\zeta_{1}\right|^{(\mu+2) / 2 \sigma} \frac{4 \sigma-\mu-v_{2}}{\sigma}\left|\zeta_{2}\right|^{\left(3 \sigma-\mu-v_{2}\right) / \sigma} \\
& \times M_{21}\left|\zeta_{1}\right|^{(\sigma-1) / \sigma} \widetilde{\eta}_{2}\left(\left|\zeta_{1}\right|^{\left(2 v_{2}+\mu\right) / 2 \sigma}\right. \\
& \left.+\left|\zeta_{2}\right|^{\left(2 v_{2}+\mu\right) / 2 \sigma}\right) \leq \frac{1}{24} \zeta_{1}^{4}+H_{242}\left(\bar{x}_{2}\right) \zeta_{2}^{4},
\end{aligned}
$$

$$
\begin{aligned}
\frac{1}{2} \frac{\partial^{2} \psi_{2}}{\partial x_{2}^{2}}\left\|g_{2}\right\|^{2} \leq & \frac{4 \sigma-\mu-v_{2}}{2 v_{2}}\left|\zeta_{2}\right|^{\left(3 \sigma-\mu-v_{2}\right) / \sigma} \\
& \cdot\left(\left|\zeta_{2}\right|^{\left(\left(\sigma-v_{2}\right) / \sigma\right)}+\lambda_{1}^{\left(\left(\sigma-v_{2}\right) / v_{2}\right)}\left|\zeta_{1}\right|^{\left(\left(\sigma-v_{2}\right) / \sigma\right)}\right) \\
& \times 2 \widetilde{\eta}_{2}^{2}\left(\left|\zeta_{1}\right|^{\left(2 v_{2}+\mu\right) / \sigma}+\left|\zeta_{2}\right|^{\left(2 v_{2}+\mu\right) / \sigma}\right) \\
\leq & \frac{1}{24} \zeta_{1}^{4}+H_{243}\left(\bar{x}_{2}\right) \zeta_{2}^{4} .
\end{aligned}
$$

Substituting equations (31)-(33) into (30), one obtains

$$
\frac{1}{2} \operatorname{tr}\left\{\bar{g}_{2}^{T} \frac{\partial^{2} \Psi_{2}}{\partial \bar{x}_{2}^{2}} \bar{g}_{2}\right\} \leq \frac{1}{8} \zeta_{1}^{4}+H_{24}\left(\bar{x}_{2}\right) \zeta_{2}^{4},
$$

where $H_{24}\left(\bar{x}_{2}\right)=H_{241}\left(\bar{x}_{2}\right)+H_{242}\left(\bar{x}_{2}\right)+H_{243}\left(\bar{x}_{2}\right) \geq 0$ is a $C^{2}$ function.

Let $H_{2}\left(\bar{x}_{2}\right)=H_{21}\left(\bar{x}_{2}\right)+H_{22}\left(\bar{x}_{2}\right)+H_{23}\left(\bar{x}_{2}\right)+H_{24}\left(\bar{x}_{2}\right)$ $\geq 0$. Design the virtual controller $\xi_{3}$ as

$$
\begin{aligned}
\xi_{3} & =-\lambda_{2}\left(\bar{x}_{2}\right)\left\lceil\zeta_{2}\right\rceil^{\left(v_{3} / \sigma\right)} \text { with } \lambda_{2}\left(\bar{x}_{2}\right) \\
& =\left(\frac{n-1+H_{2}\left(\bar{x}_{2}\right)}{\underline{\vartheta}_{2}}\right)^{1 / q_{2}}>0 .
\end{aligned}
$$

Substituting (20), (27), (29), (34), and (35) into (16), one can obtain

$$
\begin{aligned}
\ell V_{2} \leq & -\frac{1}{2} G\left(x_{1}\right) \zeta_{1}^{4}-(n-1)\left(\zeta_{1}^{4}+\zeta_{2}^{4}\right)+\vartheta_{2}(t)\left\lceil\zeta_{2}\right\rceil^{\left(4 \sigma-\mu-v_{2}\right) / \sigma} \\
& \cdot\left(x_{3}^{q_{2}}-\xi_{3}^{q_{2}}\right) .
\end{aligned}
$$

Inductive Step. Suppose at step $i-1$, there exist a $C^{2}$ Lyapunov function $V_{i-1}: \Pi_{i-1} \longrightarrow R^{+}$, and a range of continuous virtual controllers $\xi_{1}, \xi_{2}, \ldots, \xi_{i}$ defined as

$$
\begin{aligned}
& \xi_{1}=0, \\
& \zeta_{1}=\left\lceil x_{1}\right\rceil^{\sigma / v_{1}}-\left\lceil\xi_{1}\right\rceil^{\sigma / v_{1}}, \\
& \xi_{2}=-\lambda\left(x_{1}\right)\left\lceil\zeta_{1}\right\rceil^{v_{2} / \sigma}, \\
& \zeta_{2}=\left\lceil x_{2}\right\rceil^{\sigma / v_{2}}-\left\lceil\xi_{2}\right\rceil^{\sigma / v_{2}}, \\
& \xi_{3}=-\lambda_{2}\left(\bar{x}_{2}\right)\left\lceil\zeta_{2}\right\rceil^{v_{3} / \sigma}, \\
& \zeta_{3}=\left\lceil x_{3}\right\rceil^{\sigma / v_{3}}-\left\lceil\xi_{3}\right\rceil^{\sigma / v_{3}}, \\
& \vdots \\
& \xi_{i}=-\lambda_{i-1}\left(\bar{x}_{i-1}\right)\left\lceil\zeta_{i-1}\right\rceil^{v_{i} / \sigma}, \\
& \zeta_{i}=\left\lceil x_{i}\right\rceil^{\sigma / v_{i}}-\left\lceil\xi_{i}\right\rceil^{\sigma / v_{i}},
\end{aligned}
$$




$$
\begin{aligned}
\ell V_{i-1} \leq & -\frac{1}{2} G\left(x_{1}\right) \zeta_{1}^{4}-(n+2-i) \\
& \cdot \sum_{l=1}^{i-1} \zeta_{i}^{4}+\vartheta_{i-1}(t)\left\lceil\zeta_{i-1}\right\rceil^{\left(4 \sigma-\mu-v_{i}\right) / \sigma}\left(x_{i}^{q_{i-1}}-\xi_{i}^{q_{i-1}}\right) .
\end{aligned}
$$

Then, the following property can be inferred.

Proposition 1. Choose the ith Lyapunov function $V_{i}: \Pi_{i} \longrightarrow R^{+}$as $V_{i}=V_{i-1}+\Psi_{i}$ with

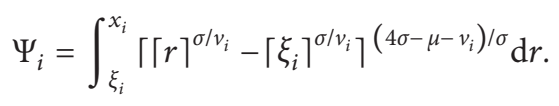

Then, $V_{i}$ is $C^{2}$ on $\Pi_{i}$ and there exists a virtual controller $\xi_{i+1}$ such that

$$
\begin{aligned}
\ell V_{i} \leq & -\frac{1}{2} G\left(x_{1}\right) \zeta_{1}^{4}-(n+1-i) \sum_{l=1}^{i} \zeta_{l}^{4}+\vartheta_{i-1}(t)\left\lceil\zeta_{i}\right\rceil^{\left(4 \sigma-\mu-v_{i}\right) / \sigma} \\
& \cdot\left(x_{i+1}^{q_{i}}-\xi_{i+1}^{q_{i}}\right)
\end{aligned}
$$

where

$$
\begin{aligned}
\xi_{i+1} & =-\lambda_{i}\left(\bar{x}_{i}\right)\left\lceil\zeta_{i}\right\rceil^{v_{i+1} / \sigma} \text { with } \lambda_{i}\left(\bar{x}_{i}\right) \\
& =\left[\frac{n-i+1+H_{i}\left(\bar{x}_{i}\right)}{\underline{\vartheta}_{i}}\right]^{1 / q_{i}}>0 .
\end{aligned}
$$

The proof of above Proposition 1 is provided in the Appendix.

Step 3. In light of the inductive step, when $i=n$ and $x_{n+1}=u$, Proposition 1 holds. Thus, we choose the overall Lyapunov function $V_{n}$ as $V_{n}=V_{n-1}+\Psi_{n}$ with

$$
\Psi_{n}=\int_{\xi_{n}}^{x_{n}}\left\lceil\lceil r\rceil^{\sigma / v_{n}}-\left\lceil\xi_{n}\right\rceil^{\sigma / v_{n}}\right\rceil^{\left(4 \sigma-\mu-v_{n}\right) / \sigma} \mathrm{d} r
$$

and define the virtual controller $\xi_{n+1}$ as

$$
\xi_{n+1}=-\lambda_{n}(x)\left\lceil\varsigma_{n}\right\rceil^{v_{n}+1 / \sigma} \text { with } \lambda_{n}(x)=\left[\frac{1+H_{n}(x)}{\underline{\vartheta}_{n}}\right]^{1 / q_{n}}>0 \text {. }
$$

Then, $V_{n}(x)$ is clearly a $C^{2}$ function on $\Pi_{n}$, and it is easy to obtain that

$$
\ell V_{n} \leq-\frac{1}{2} G\left(x_{1}\right) \zeta_{l}^{4}-\sum_{l=1}^{n} \zeta_{l}^{4}+\vartheta_{n}(t)\left\lceil\zeta_{n}\right\rceil^{\left(4 \sigma-\mu-v_{n}\right) / \sigma}\left(u^{q_{n}}-\xi_{n+1}^{q_{n}}\right)
$$

Therefore, we can design

$$
\begin{aligned}
& u=\xi_{n+1}(x)=-\lambda_{n}(x)\left\lceil\left\lceil x_{n}\right\rceil^{\sigma / v_{n}}\right. \\
& +\lambda_{n-1}^{\sigma / v_{n}}\left\lceil x_{n-1}\right\rceil^{\sigma / v_{n-1}}+\lambda_{n-1}^{\sigma / v_{n}} \lambda_{n-2}^{\sigma / v_{n-2}}\left\lceil x_{n-2}\right\rceil^{\sigma / v_{n-2}} \\
& \left.+\cdots+\lambda_{n-1}^{\sigma / v_{n}} \lambda_{n-2}^{\sigma / v_{n-1}} \cdots \lambda_{1}^{\sigma / v_{2}}\left\lceil x_{1}\right\rceil^{\sigma / v_{1}}\right\rceil^{v_{n}+\mu / \sigma q n} \text {, }
\end{aligned}
$$

which results in

$$
\ell V_{n} \leq-\frac{1}{2} G\left(x_{1}\right) \zeta_{1}^{4}-\sum_{l=1}^{n} \zeta_{l}^{4} \leq 0 .
$$

3.2.2. Part II: Verification of Keeping the Output Constraint. For any $x(0)=\left(x_{1}(0)\right)^{T} \in \Pi_{n}$, by Ito's formula and (46), we can deduce

$$
\begin{aligned}
0 \leq E V_{n}(x(t)) & =V_{n}(x(0))+E \int_{0}^{t} \ell V_{n}(x(\tau)) d r \\
& \leq V_{n}(x(0))<\infty .
\end{aligned}
$$

From $V_{n}(x)=V_{1}\left(x_{1}\right)+\sum_{l=2}^{n} \Psi_{l}>0$ and (47), it can be further verified that

$$
0 \leq E V_{1}\left(x_{1}(t)\right) \leq V_{n}\left(x_{0}\right)<\infty,
$$

which indicates

$$
P\left\{V_{1}\left(x_{1}(t)\right)<\infty\right\}=1 .
$$

Hence, $P\{|y(t)|<b\}=P\left\{\left|x_{1}(t)\right|<b\right\}=1$. Then, Part I of Theorem 1 is proved.

3.2.3. Part III: Stability Analysis. Based on the definition of $V_{n}$, we easily obtain the fact that $V_{n}$ is radially unbounded. Combining the fact with (40) and Lemma 1 directly infers that system (1) has a solution $x(t, x(0))$ for any $x(0) \in \Pi_{n}$.

On the contrary, by a simple calculation, one obtains

$$
\begin{aligned}
& V_{n}=V_{1}+\sum_{l=2}^{n} \Psi_{l} \\
& =V_{1}+\sum_{l=2}^{n} \int_{\xi_{l}}^{x_{l}}\left\lceil\lceil r\rceil^{\frac{\sigma}{v_{l}}}-\left\lceil\xi_{l}\right\rceil^{\left.\frac{\sigma}{v_{l}}\right\rceil}\left(4 \sigma-\mu-v_{l}\right) / \sigma d \mathrm{~d} r\right. \\
& \leq \frac{2 b^{4 \sigma-\mu}}{(4 \sigma-\mu) \pi} \tan \left(\frac{\pi\left|x_{1}\right|^{4 \sigma-\mu}}{2 b^{4 \sigma-\mu}}\right)+2 \sum_{l=2}^{n}\left|\zeta_{l}\right|^{(4 \sigma-\mu) / \sigma} .
\end{aligned}
$$

In addition, since $4 \sigma-\mu>1$, it is easy to get that $0 \leq\left(\pi\left|x_{1}\right|^{4 \sigma-\mu} / 2 b^{4 \sigma-\mu}\right)<(\pi / 2)$ for all $x_{1} \in \Pi_{1}$. Then, applying the characteristics of tangent functions, it is not difficult to infer that 


$$
\begin{aligned}
\tan \left(\frac{\pi\left|x_{1}\right|^{4 \sigma-\mu}}{2 b^{4 \sigma-\mu}}\right) & \leq \frac{\pi}{2 b^{4 \sigma-\mu}}\left|x_{1}\right|^{4 \sigma-\mu} G\left(x_{1}\right) \\
& \leq \frac{\pi(4 \sigma-\mu)}{2 b^{4 \sigma-\mu}}\left|x_{1}\right|^{4 \sigma-\mu} G\left(x_{1}\right) .
\end{aligned}
$$

Now, let $\quad c=2^{(\mu-8 \sigma) /(4 \sigma-\mu) 4}>0 \quad$ and $0<\gamma=(4 \sigma /(4 \sigma-\mu))<1$. According to (44), (45), and Lemma 4 , we obtain

$$
\begin{aligned}
c V_{n}^{\gamma} \leq & 2^{(\mu-8 \sigma) /(4 \sigma-\mu)}\left[\frac{2 b^{4 \sigma-\mu}}{(4 \sigma-\mu) \pi} \tan \left(\frac{\pi\left|x_{1}\right|^{4 \sigma-\mu}}{2 b^{4 \sigma-\mu}}\right)+2 \sum_{l=1}^{n}\left|\zeta_{1}\right|^{4 \sigma-\mu}\right]^{4 \sigma /(4 \sigma-\mu)} \\
\leq & 2^{(\mu-8 \sigma) /(4 \sigma-\mu)} G\left(x_{1}\right)^{4 \sigma /(4 \sigma-\mu)} \varsigma_{1}^{4} \\
& +\frac{1}{2} \sum_{l=1}^{n} \zeta_{1}^{4} \leq \frac{1}{2} G\left(x_{1}\right) \zeta_{1}^{4}+\frac{1}{2} \sum_{l=1}^{n} \zeta_{1}^{4} .
\end{aligned}
$$

So, one further obtains

$$
\begin{aligned}
\ell V_{n}+c V_{n}^{\gamma}= & \ell V_{n}+2^{(\mu-8 \sigma) /(4 \sigma-\mu)} V_{n}^{4 \sigma /(4 \sigma-\mu)} \\
\leq & -\frac{1}{2} G\left(x_{1}\right) \zeta_{1}^{4}-\sum_{l=1}^{n} \varsigma_{1}^{4} \\
& +\frac{1}{2} G\left(x_{1}\right) \zeta_{1}^{4}+\frac{1}{2} \sum_{l=1}^{n} \zeta_{1}^{4} \leq-\frac{1}{2} \sum_{l=1}^{n} \zeta_{1}^{4} \leq 0 .
\end{aligned}
$$

In other words, $\ell V_{n} \leq-c V_{n}^{\gamma}$. Consequently, it is directly deduced from Lemma 2 that the origin of system (1) under controller (39) is finite-time stable in probability.

Remark 3. Comparing with the existing results in most of the literatures, the paper mainly focuses on the finite-time stabilization, instead of the boundness of tracking error. Moreover, the proposed approach can be extend to the tracking control by introducing a coordinate transformation before constructing the BLF.

\section{Simulation}

In this section, we will provide the simulation results of the following example to illustrate the validity of the proposed strategy:

$$
\left\{\begin{array}{l}
\mathrm{d} x_{1}=x_{2}^{7 / 5} \mathrm{~d} t \\
\mathrm{~d} x_{2}=u \mathrm{~d} t+\frac{1}{2} x_{1}^{2} x_{2}^{1 / 5} \mathrm{~d} t+\frac{1}{8}\left(\sin x_{2}\right)^{2} x_{2}^{3 / 5} \mathrm{~d} \omega \\
y=x_{1}
\end{array}\right.
$$

where $q_{1}=7 / 5, q_{2}=1$, and $\mu=-4 / 11 \in(-(5 / 12), 0)$. Then, $v_{1}=1, v_{2}=5 / 11$, and $v_{3}=1 / 11$. Since $\vartheta_{1}(t)=\vartheta_{2}(t)=1$,

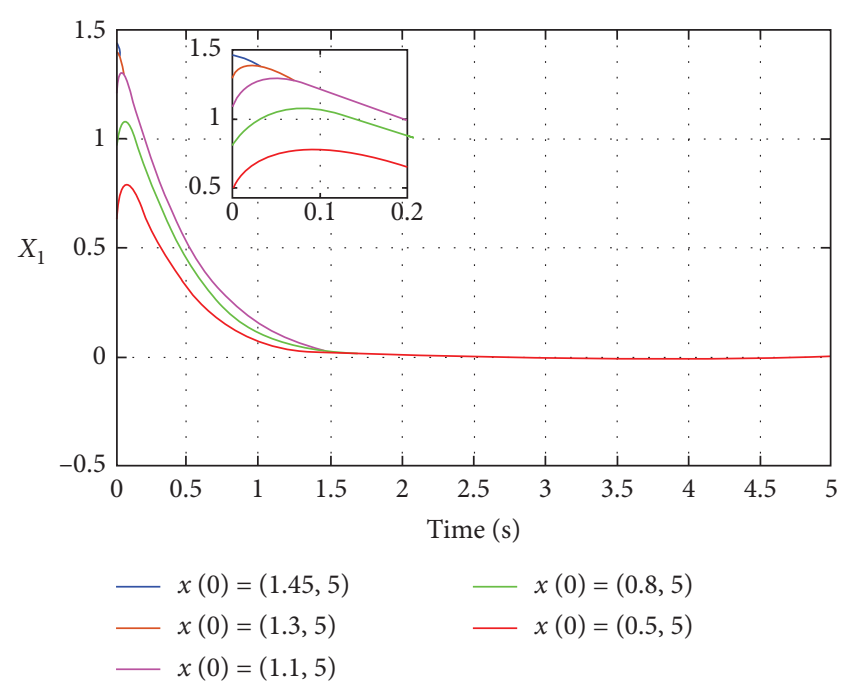

Figure 1: Trajectories of $x_{1}(t)$.

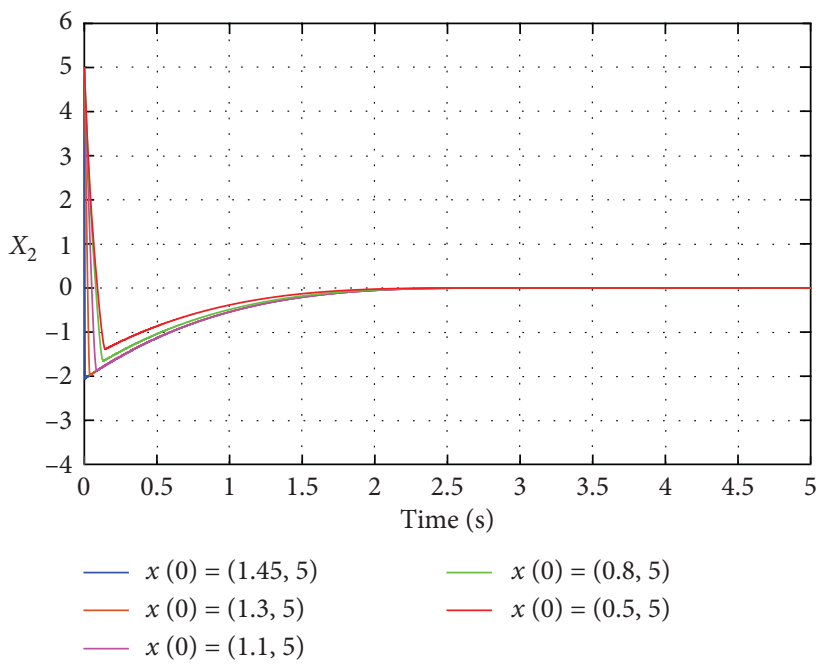

Figure 2: Trajectories of $x_{2}(t)$.

Assumption 1 is satisfied. And Assumption 2 is also satisfied with $\psi_{1}=\eta_{1}=0, \quad \psi_{2}=(1 / 2) x_{1}^{2}$, and $\eta_{2}=(1 / 8)\left(\sin x_{2}\right)^{2}$. Furthermore, note that $c_{1}=1, c_{2}=11 / 5>2$, and $\sigma_{0}=1$. Thus, one can select $\sigma=\sigma_{0}=1$.

Now, let $\varsigma_{1}=\left\lceil x_{1}\right\rceil$ and $G\left(x_{1}\right)=\sec ^{2}\left(\pi\left|x_{1}\right|^{48 / 11} / 2 b^{48 / 11}\right)$. In view of design procedure in Part I of the proof, we can design the virtual controller $\xi_{2}$ as

$$
\xi_{2}=-2^{5 / 7}\left\lceil\zeta_{1}\right\rceil^{5 / 11}:=-\lambda_{1}\left\lceil\zeta_{1}\right\rceil^{5 / 11} .
$$

Next, according to the design procedure, we denote $\varsigma_{2}=$ $\left\lceil x_{2}\right]^{11 / 5}-\left\lceil\xi_{2}\right\rceil^{11 / 5}$ and further obtain that 


$$
\begin{aligned}
& H_{21}=\frac{7}{44}\left(2 G\left(x_{1}\right)\right)^{44 / 7}\left(\frac{44}{296}\right)^{-37 / 7}, \\
& H_{22}=\frac{86}{11} \lambda_{1}^{11 / 5}+\frac{37}{44}\left(\frac{86}{11} \lambda_{1}^{18 / 5}\right)^{44 / 37}\left(\frac{44}{56}\right)^{-7 / 37}, \\
& H_{23}=\frac{1}{2} x_{1}^{2} \lambda_{1}^{1 / 7}+\frac{43}{44}\left(\frac{1}{2} x_{1}^{2} \lambda_{1}^{1 / 7}\right)^{44 / 43}\left(\frac{44}{8}\right)^{-1 / 43}, \\
& H_{24}=\frac{37}{320}\left(\sin x_{2}\right)^{4}+\frac{32}{44}\left(\frac{37}{320}\left(\sin x_{2}\right)^{4} \lambda_{1}^{12 / 5}\right)^{44 / 32}\left(\frac{44}{96}\right)^{-12 / 32} .
\end{aligned}
$$

Let $H_{2}=H_{21}+H_{22}+H_{23}+H_{24}$ and $\lambda_{2}=1+H_{2}$; then, the controller $u$ can be designed as

$$
u=-\lambda_{2}\left[\zeta_{2}\right]^{1 / 11}=-\lambda_{2}\left[\left[x_{2}\right]^{11 / 5}-\left[\xi_{2}\right]^{11 / 5}\right]^{1 / 11}
$$

Finally, we suppose $b=1.5$ and select some different initial states $x(0)$ 's with each $x(0)$ satisfying $x(0) \in \Pi_{2}$. The simulation results of system (48) are shown in Figures 1 and 2. Figure 1 curves trajectories of $x_{1}(t)$ under different initial values, which illustrate that the output constraint is always not violated. Meanwhile, the trajectories of $x_{2}(t)$ is given in Figure 2. It can be observed from the two figures that system (48) under controller (51) is finite-time stable.

\section{Conclusion}

In this paper, the stability issue is addressed for a class of $p$-norm stochastic systems with output constraints and unknown time-varying parameters. Using a tan-type BLF, the finite-time control strategy is proposed by the adding a power integrator technique. On this basis, the designed controller has been proved to ensure that the origin of the closed-loop system is finite-time stable in probability and the system output is kept in a pre-given set. This conclusion has also been verified by the simulation results. It should be pointed out that the proposed approach is not applicable to the case of asymmetrical output constraints. In the future, we will try to modify the proposed method to be suitable for stochastic systems with asymmetrical output constraints or multi-input multi-output stochastic systems.

\section{Appendix}

Proof of Proposition 1. For $j, k=1, \ldots, i-1$, one can get from the definition of $\Psi_{i}$ that

$$
\begin{aligned}
\frac{\partial \Psi_{i}}{\partial x_{i}}= & \left\lceil\varsigma_{i}\right\rceil^{\left(4 \sigma-\mu-v_{i}\right) / \sigma}, \\
\frac{\partial \Psi_{i}}{\partial x_{j}}= & \frac{-4 \sigma+\mu+v_{i}}{\sigma} \frac{\partial\left\lceil\xi_{i}\right\rceil^{\sigma / v_{i}}}{\partial x_{j}} \int_{\xi_{i}}^{x_{i}}\left|\lceil r\rceil^{\sigma / v_{i}}-\left\lceil\xi_{i}\right\rceil^{\sigma / v_{i}}\right|^{\left(3 \sigma-\mu-v_{i}\right) / \sigma} \mathrm{d} r, \\
\frac{\partial^{2} \Psi_{i}}{\partial x_{j} \partial x_{i}}= & \frac{-4 \sigma+\mu+v_{i}}{\sigma}\left|\varsigma_{i}\right|^{\left(3 \sigma-\mu-v_{i}\right) / \sigma} \frac{\partial\left\lceil\xi_{i}\right\rceil^{\sigma / v_{i}}}{\partial x_{j}}, \\
\frac{\partial^{2} \Psi_{i}}{\partial x_{j} \partial x_{k}}= & \frac{\partial^{2} \Psi_{i}}{\partial x_{k} \partial x_{j}}=\frac{-4 \sigma+\mu+v_{i}}{\sigma} \frac{\partial^{2}\left\lceil\xi_{i}\right\rceil^{\sigma / v_{i}}}{\partial x_{k} \partial x_{j}} \int_{\xi_{i}}^{x_{i}}\left|\lceil r\rceil^{\sigma / v_{i}}-\left\lceil\xi_{i}\right\rceil^{\sigma / v_{i} \mid}\right|^{\left(3 \sigma-\mu-v_{i}\right) / \sigma} \mathrm{d} r \\
& +\frac{4 \sigma-\mu-v_{i}}{\sigma \sigma-\mu-v_{i}} \frac{\partial\left\lceil\xi_{i}\right\rceil^{\sigma / v_{i}}}{\partial x_{j}} \frac{\partial\left\lceil\xi_{i}\right\rceil^{\sigma / v_{i}}}{\partial x_{k}} \int_{\xi_{i}}^{x_{i}}\left|\lceil r\rceil^{\sigma / v_{i}}-\left\lceil\xi_{i}\right\rceil^{\sigma / v_{i} \mid}\right|^{\left(2 \sigma-\mu-v_{i}\right) / \sigma} \mathrm{d} r, \\
\frac{\partial^{2} \Psi_{i}}{\partial x_{i}^{2}}= & \frac{4 \sigma-\mu-v_{i}}{v_{i}}\left|x_{i}\right|^{\left(\sigma-v_{i}\right) / v_{i}}\left|\varsigma_{i}\right|^{\left(3 \sigma-\mu-v_{i}\right) / \sigma} .
\end{aligned}
$$


Then, we have

$$
\begin{aligned}
\ell V_{i}= & \ell V_{i-1}+\sum_{j=1}^{i-1} \frac{\partial \Psi_{i}}{\partial x_{j}}\left(\vartheta_{j}(t) x_{j+1}^{q_{j}}+f_{j}\right)+\frac{\partial \Psi_{i}}{\partial x_{j}}\left(\vartheta_{i}(t) x_{i+1}^{q_{i}}+f_{i}\right) \\
& +\frac{1}{2} \operatorname{tr}\left\{\bar{g}_{i}^{T} \frac{\partial^{2} \Psi_{i}}{\partial \bar{x}_{i}^{2}} \bar{g}_{i}\right\} \\
\leq & -\frac{1}{2} G\left(x_{1}\right) \zeta_{1}^{4}-(n+2-i) \sum_{l=1}^{i-1} \zeta_{l}^{4}+\vartheta_{i-1}(t) \\
& \cdot\left[\zeta_{i-1}\right]\left(4 \sigma-\mu-v_{i-1}\right) / \sigma\left(x_{j}^{q_{i-1}}-\xi_{i}^{q_{i-1}}\right) \\
& +\sum_{j=1}^{i-1} \frac{\partial \Psi_{i}}{\partial x_{j}}\left(\vartheta_{j}(t) x_{j+1}^{q_{j}}+f_{j}\right)+\frac{\partial \Psi_{i}}{\partial x_{i}} \vartheta_{i}(t) x_{i+1}^{q_{i}}+\frac{\partial \Psi_{i}}{\partial x_{i}} f_{i} \\
& +\frac{1}{2} \operatorname{tr}\left\{\bar{g}_{i}^{T} \frac{\partial^{2} \Psi_{i}}{\partial \bar{x}_{i}^{2}} \bar{g}_{i}\right\} .
\end{aligned}
$$

In the following, we introduce some sub-propositions to simplify the proof.

Proposition A.1. For $i=3, \ldots, n$, there exist smooth nonnegative functions $\widetilde{\psi}_{i}(\cdot)$ and $\widetilde{\eta}_{i}(\cdot)$ such that

$$
\begin{aligned}
\left|f_{i}\left(\bar{x}_{i}\right)\right| & \leq \widetilde{\psi}_{i}\left(\bar{x}_{i}\right)\left(\left|\zeta_{1}\right|^{\left(v_{i}+\mu\right) / \sigma}+\cdots+\left|\zeta_{i}\right|^{\left(v_{i}+\mu\right) / \sigma}\right), \\
\left\|g_{i}\left(\bar{x}_{i}\right)\right\| & \leq \widetilde{\eta}_{i}\left(\bar{x}_{i}\right)\left(\left|\zeta_{1}\right|^{\left(2 v_{i}+\mu\right) / 2 \sigma}+\cdots+\left|\zeta_{i}\right|^{\left(2 v_{i}+\mu\right) / 2 \sigma}\right) .
\end{aligned}
$$

Proof. For $i=3, \ldots, n, j=1, \ldots, i$, one has

$$
\begin{aligned}
\left|x_{j}\right|^{\left(v_{i}+\mu\right) / v_{j}}= & \left|\zeta_{j}+\left\lceil\xi_{i}\right\rceil^{\frac{\sigma}{v_{j}}}\right| v^{\left(v_{i}+\mu\right) / \sigma} \leq\left|\zeta_{j}\right|^{\left(v_{i}+\mu\right) / \sigma} \\
& +\left|\lambda_{j-1}^{\sigma / v_{j}}\left\lceil\zeta_{j-1}\right\rceil\right|^{\left(v_{i}+\mu\right) / \sigma} \leq\left|\zeta_{j}\right|^{\left(v_{i}+\mu\right) / \sigma} \\
& +\lambda_{j-1}^{\left(v_{i}+\mu\right) / v_{j}}\left|\zeta_{j-1}\right|^{\left(v_{i}+\mu\right) / \sigma}, \\
\left|x_{j}\right|^{\left(2 v_{i}+\mu\right) / 2 v_{j}}= & \left|\zeta_{j}+\left\lceil\xi_{i}\right\rceil^{\frac{\sigma}{v_{j}}}\right|^{\left(2 v_{i}+\mu\right) / 2 \sigma} \leq\left|\zeta_{j}\right|^{\left(2 v_{i}+\mu\right) / 2 \sigma} \\
& +\left|\lambda_{j-1}^{\sigma / v_{j}}\left\lceil\zeta_{j-1}\right\rceil\right|^{\left(2 v_{i}+\mu\right) / 2 \sigma} \leq\left|\zeta_{j}\right|^{\left(2 v_{i}+\mu\right) / 2 \sigma} \\
& +\left|\lambda_{j-1}^{\left(2 v_{i}+\mu\right) / 2 v_{j}}\right| \zeta_{j-1}||^{\left(2 v_{i}+\mu\right) / 2 \sigma},
\end{aligned}
$$

which means

$$
\begin{aligned}
& \left|f_{i}\left(\bar{x}_{i}\right)\right| \leq \psi_{i}\left(\bar{x}_{i}\right)\left[\left|\varsigma_{1}\right|^{\left(v_{i}+\mu\right) / \sigma}+\sum_{j=2}^{i}\left(\left|\varsigma_{j}\right|^{\left(v_{i}+\mu\right) / \sigma}+\lambda_{j-1}^{\left(v_{i}+\mu\right) / v_{j}}\left|\varsigma_{j-1}\right|{ }^{\left(v_{i}+\mu\right) / \sigma}\right)\right] \leq \widetilde{\psi}_{i}\left(\bar{x}_{i}\right) \sum_{j=1}^{i}\left|\varsigma_{j}\right|^{\left(v_{i}+\mu\right) / \sigma} \\
& \left\|g_{i}\left(\bar{x}_{i}\right)\right\| \leq \eta_{i}\left(\bar{x}_{i}\right)\left[\left|\varsigma_{1}\right|^{\left(2 v_{i}+\mu\right) / 2 \sigma}+\sum_{j=2}^{i}\left(\left|\varsigma_{j}\right|^{\left(2 v_{i}+\mu\right) / 2 \sigma}+\lambda_{j-1}^{\left(2 v_{i}+\mu\right) / 2 v_{j}}\left|\varsigma_{j-1}\right|^{\left(2 v_{i}+\mu\right) / 2 \sigma}\right)\right] \leq \widetilde{\eta}_{i}\left(\bar{x}_{i}\right) \sum_{j=1}^{i}\left|\varsigma_{j}\right|^{\left(2 v_{i}+\mu\right) / 2 \sigma}
\end{aligned}
$$

where $_{\left(2 v_{i}+\mu\right) / 2 v_{j}} \widetilde{\psi}_{i}(\cdot) \geq\left[\sum_{j=1}^{i}\left(1+\lambda_{j-1}^{\left(v_{i}+\mu\right) / v_{j}}\right)\right] \psi_{i}(\cdot), \widetilde{\eta}_{i}(\cdot) \geq\left[\sum_{j=1}^{i}(1+\right.$ $\left.\left.\lambda_{j-1}^{\left(2 v_{i}+\mu\right) / 2 v_{j}}\right)\right] \eta_{i}(\cdot)$ are nonnegative smooth functions.

Proposition A.2. For $i=3, \ldots, n$, there exist nonnegative $C^{2}$ functions $H_{i 1}(\cdot)$ such that

$$
\vartheta_{i-1}(t)\left[\zeta_{i-1}\right]^{\left(4 \sigma-\mu-v_{i-1}\right) / \sigma}\left(x_{i}^{q_{i-1}}-\xi_{i}^{q_{i-1}}\right) \leq \frac{1}{8} \zeta_{i-1}^{4}+H_{i 1}\left(\bar{x}_{i}\right) \zeta_{i}^{4}
$$

Proof. Since $\left(v_{i-1}+\mu\right) / \sigma \leq 1$, it can be gotten from Lemma 5 that

$$
\begin{aligned}
\left|x_{i}^{q_{i-1}}-\xi_{i}^{q_{i-1}}\right| & \leq 2^{1-\left(\left(v_{i-1}+\mu\right) / \sigma\right)}\left|\left[x_{i}\right]^{\sigma / v_{i}}-\left[\xi_{i}\right]^{\sigma / v_{i}}\right|^{\left(\left(v_{i-1}+\mu\right) / \sigma\right)} \\
& \leq 2\left|\zeta_{i}\right|^{\left(\left(v_{i-1}+\mu\right) / \sigma\right)}
\end{aligned}
$$

From (A.7) and Lemma 3, one can verify that

$$
\begin{aligned}
& \vartheta_{i-1}(t)\left[\varsigma_{i-1}\right]^{\left(4 \sigma-\mu-v_{i-1}\right) / \sigma}\left(x_{i}^{q_{i}-1}-\xi_{i}^{q_{i}-1}\right) \\
& \quad \leq 2 \bar{\vartheta}_{i-1}\left|\varsigma_{i-1}\right|^{\left(4 \sigma-\mu-v_{i-1}\right) / \sigma}\left|\varsigma_{i}\right|^{\left(v_{i-1}+\mu\right) / \sigma} \leq \frac{1}{8} \varsigma_{i-1}^{4}+H_{i 1}\left(\bar{x}_{i}\right) \varsigma_{i}^{4},
\end{aligned}
$$

where $H_{i 1}(\cdot) \geq 0$ is a $C^{2}$ function.

Proposition A.3. For $i=3, \ldots, n$, there exist nonnegative $C^{2}$ functions $H_{i 2}(\cdot)$ such that

$$
\sum_{j=1}^{i-1} \frac{\partial \Psi}{\partial x_{j}}\left(\vartheta_{j}(t) x_{j-1}^{q_{j}}+f_{j}\right) \leq \frac{1}{8} \sum_{l=4}^{i-1} \zeta_{l}^{4}+H_{i 2}\left(\bar{x}_{i}\right) \zeta_{i}^{4} .
$$

Proof. For $i=3, \ldots, n, j=1, \ldots, i-1$, there are nonnegative $C^{2}$ functions $\widehat{M}_{i j}(\cdot), M_{i j}(\cdot)$ such that 


$$
\begin{aligned}
\left|\frac{\partial\left[\xi_{i}\right]^{\sigma / v_{i}}}{\partial x_{j}}\right|= & \left|\frac{\partial \lambda_{i-1}^{\sigma / v_{i}}\left(\bar{x}_{i-1}\right)}{\partial x_{j}}\right|\left|\zeta_{i-1}\right|+\lambda_{i-1}^{\sigma / v_{i}}\left(\bar{x}_{i-1}\right)\left|\frac{\partial \lambda_{i-2}^{\sigma / v_{i-1}}\left(\bar{x}_{i-2}\right)}{\partial x_{j}}\right|\left|\zeta_{i-2}\right|+\ldots+\lambda_{i-1}^{\sigma / \tau_{i}}\left(\bar{x}_{i-1}\right) \times \lambda_{i-2}^{\sigma /\left(\tau_{i-1}\right)}\left(\bar{x}_{i-2}\right) \times \cdots \\
& \times \lambda_{j+1}^{\sigma / v_{j+2}}\left(\bar{x}_{j+1}\right)\left|\frac{\partial \lambda_{j}^{\sigma /\left(v_{i+1}\right)}\left(\bar{x}_{j}\right)}{\partial x_{j}}\right| \zeta_{j}\left|+\lambda_{i-1}^{\sigma / v_{i}}\left(\bar{x}_{i-1}\right) \times \lambda_{i-2}^{\sigma /\left(v_{i-1}\right)}\left(\bar{x}_{i-2}\right) \times \cdots \times \lambda_{j+1}^{\sigma / v_{j+2}}\left(\bar{x}_{j-1}\right) \lambda_{j}^{\sigma / v_{j+1}}\left(\bar{x}_{j}\right) \times \frac{\sigma}{v_{j}}\right| x_{i} \mid{ }^{\left(\sigma / v_{j}\right)-1} \\
\leq & \widehat{M}_{i j}\left(\bar{x}_{i}\right)\left[\sum_{\tau=j}^{i-1}\left|\varsigma_{\tau}\right|+\left|\varsigma_{j}\right|^{1-\left(v_{j} / \sigma\right)}+\left|\varsigma_{j-1}\right|^{1-\left(v_{j} / \sigma\right)} \lambda_{j-1}^{\sigma / v_{j}-1}\left(\bar{x}_{j-1}\right)\right] \leq M_{i j}\left(\bar{x}_{i}\right) \sum_{l=j-1}^{i-1}\left|\varsigma_{j}\right|^{1-\left(v_{j} / \sigma\right)} .
\end{aligned}
$$

Then, from (A.10), we can get

$$
\begin{aligned}
\frac{\partial \Psi_{i}}{\partial x_{i}}\left(\vartheta_{j}(t) x_{j+1}^{q_{j}}+f_{j}\right) \leq & \frac{4 \sigma-v_{i}-\mu}{\sigma} \overline{\mathcal{Q}}_{j}\left|\varsigma_{i}\right|^{\left(\left(4 \sigma-v_{i}-\mu\right) / \sigma\right)-1} \times M_{i j}\left(\bar{x}_{i}\right) \\
& \times\left[\sum_{\tau=j-1}^{i-1}\left|\varsigma_{T}\right|^{1-\left(v_{j} / \sigma\right)}\left|x_{i}-\xi_{i}\right|\right]\left[\left|x_{j+1}\right|^{\left(v_{j}+\mu\right) /\left(v_{j}+1\right)}+\widetilde{\psi}_{j} \sum_{l=1}^{j}\left|\varsigma_{l}\right|^{\left(v_{j}+\mu\right) / \sigma}\right] \leq \frac{1}{8(i-1)} \sum_{l=1}^{i-1} \varsigma_{l}^{4}+H_{i 2 j}\left(\bar{x}_{i}\right) \varsigma_{i}^{4},
\end{aligned}
$$

where $\widetilde{M}_{i j}(\cdot), h_{i l}(\cdot)$ and $H_{i 2 j}(\cdot)$ are nonnegative $C^{2}$ functions. Let $H_{i 2}(\cdot)=\sum_{j=1}^{i-1} H_{i 2 j}(\cdot)$, which directly verify (A.9).

Proposition A.4. For $i=3, \ldots, n, j=1, \ldots, i-1$, there exist nonnegative $C^{2}$ functions $H_{i 3}(\cdot)$ such that

$$
\frac{\partial \Psi_{i}}{\partial x_{i}} f_{i} \leq \frac{1}{8} \sum_{l}^{i-1} \zeta_{l}^{4}+H_{i 3}\left(\bar{x}_{i}\right) \zeta_{i}^{4}
$$

Proof. For $i=3, \ldots, n$, according to Proposition A.1 and Lemma 3, there exist nonnegative $C^{2}$ functions $H_{i 3}(\cdot)$ such that

$$
\begin{aligned}
\frac{\partial \Psi_{i}}{\partial x_{i}} f_{i} & \leq\left|\varsigma_{i}\right|^{\left(4 \sigma-\mu-v_{i}\right) / \sigma} \times \widetilde{\psi}_{i}\left(\bar{x}_{i}\right) \sum_{j=1}^{i}\left|\varsigma_{i}\right|^{\left(v_{i}+\mu\right) / \sigma} \\
& \leq \frac{1}{8} \sum_{l=1}^{i-1} \varsigma_{l}^{4}+H_{i 3}\left(\bar{x}_{i}\right) \varsigma_{i}^{4} .
\end{aligned}
$$

Proposition A.5. For $i=3, \cdots, n$, there exist nonnegative $C^{2}$ functions $H_{i 4}(\cdot)$ such that

$$
\frac{1}{2} \operatorname{tr}\left\{\bar{g}_{i}^{T} \frac{\partial^{2} \Psi_{i}}{\partial \bar{x}_{i}^{2}} \bar{g}_{i}\right\} \leq \frac{1}{8} \sum_{l=1}^{i-1} \zeta_{l}^{4}+H_{i 2}\left(\bar{x}_{i}\right) \zeta_{i}^{4}
$$

Proof. Note that

$$
\begin{aligned}
\frac{1}{2} \operatorname{tr}\left\{\bar{g}_{i}^{T} \frac{\partial^{2} \Psi_{i}}{\partial \bar{x}_{i}^{2}} \bar{g}_{i}\right\}= & \frac{1}{2}\left\{\sum_{k, j=1, k \neq j}^{i-1} \frac{\partial^{2} \Psi_{i}}{\partial x_{k} \partial x_{j}} g_{k}^{T} g_{j}+\sum_{k=1}^{i=1} \frac{\partial^{2} \Psi_{i}}{\partial x_{k}^{2}}\left\|g_{k}\right\|^{2}\right\} \\
& +\left\{2 \sum_{j=1}^{i-1} \frac{\partial^{2} \Psi_{i}}{\partial x_{j} \partial x_{i}} g_{j}^{T} g_{i}+\frac{\partial^{2} \Psi_{i}}{\partial x_{i}^{2}}\left\|g_{i}\right\|^{2}\right\} .
\end{aligned}
$$

First of all, one gets from (A.10) that

$$
\begin{aligned}
\left|\frac{\partial^{2}\left[\xi_{i}\right]^{\sigma / v_{i}}}{\partial x_{k}^{2}}\right| \leq\left|\frac{\partial M_{i k}\left(\bar{x}_{i}\right)}{\partial x_{k}}\right| \sum_{l=1}^{i-1}\left|\zeta_{l}\right|^{1-\left(v_{k} / \sigma\right)} \\
+M_{i k}\left(\bar{x}_{i}\right) \frac{\sigma-v_{k}}{\sigma}\left|\zeta_{k}\right|^{-\left(v_{k} / \sigma\right)} \frac{\sigma}{v_{k}}\left|x_{k}\right|^{\sigma / v_{k}-1} \\
+M_{i k}\left(\bar{x}_{i}\right) \frac{\sigma-v_{k}}{\sigma} \sum_{l=k+1}^{i-1}\left|\zeta_{l}\right|^{-\left(v_{k} / \sigma\right)} M_{l k}\left(\bar{x}_{i}\right) \\
\\
+\sum_{q=k-1}^{l-1}\left|\zeta_{q}\right|^{1-\left(v_{k} / \sigma\right)} \leq K_{i k}\left(\bar{x}_{i}\right) \sum_{l=1}^{i-1}\left|\zeta_{l}\right|^{1-\left(2 v_{k} / \sigma\right)},
\end{aligned}
$$

where $K_{i k}(\cdot) \geq 0$ is a $C^{2}$ function.

From equation (A.10) and Proposition A.1, it can be inferred that

$$
\frac{1}{2} \frac{\partial^{2} \Psi_{i}}{\partial x_{k}^{2}}\left\|g_{k}\right\|^{2} \leq \frac{1}{32(i-1)} \sum_{l=1}^{i-1} \zeta_{l}^{4}+\widetilde{H}_{i 4 k}\left(\bar{x}_{i}\right) \zeta_{l}^{4},
$$


where $\widetilde{H}_{i 4 k}(\cdot) \geq 0$ is a $C^{2}$ function.

Then, one gets

$$
\frac{1}{2} \sum_{k=1}^{i-1} \frac{\partial^{2} \Psi_{i}}{\partial x_{k}^{2}}\left\|g_{k}\right\|^{2} \leq \frac{1}{32} \sum_{l=1}^{i-1} \zeta_{l}^{4}+H_{i 41}\left(\bar{x}_{i}\right) \zeta_{i}^{4},
$$

where $H_{i 41}(\cdot)=\sum_{k=1}^{i-1} \widetilde{H}_{i 4 k}(\cdot)$.

Besides, if $k \neq j, k>j$, there exist $C^{2}$ functions $\widetilde{K}_{i k j}(\cdot) \geq 0$ such that

$$
\begin{aligned}
\left|\frac{\partial^{2}\left[\xi_{i}\right]^{\sigma / v_{i}}}{\partial x_{k} \partial x_{j}}\right| \leq\left|\frac{\partial \widehat{M}_{i j}\left(\bar{x}_{i}\right)}{\partial x_{k}}\right|\left[\sum_{l=j}^{i-1}\left|\zeta_{l}\right|+\left|x_{j}\right|^{\left(\sigma / v_{j}\right)-1}\right] \\
+\widehat{M}_{i j}\left(\bar{x}_{i}\right) \sum_{l=k}^{i-1}\left|\frac{\partial \zeta_{l}}{\partial x_{k}}\right| \leq \widetilde{K}_{i k j}\left(\bar{x}_{i}\right) \\
\\
\cdot\left[\sum_{l=1}^{i-1}\left|\zeta_{l}\right|+\left|x_{j}\right|^{\left(\sigma / v_{j}\right)-1}+\left|x_{k}\right|^{\left(\sigma / v_{k}\right)-1}\right] .
\end{aligned}
$$

Meanwhile, if $k \neq j, k<j$, we have

$$
\begin{aligned}
\left|\frac{\partial^{2}\left[\xi_{i}\right]^{\sigma / v_{i}}}{\partial x_{k} \partial x_{j}}\right|= & \left|\frac{\partial^{2}\left[\xi_{i}\right]^{\sigma / v_{i}}}{\partial x_{j} \partial x_{k}}\right| \leq \widetilde{K}_{i j k}\left(\bar{x}_{i}\right) \\
& \cdot\left[\sum_{l=1}^{i-1}\left|\zeta_{l}\right|+\left|x_{k}\right|^{\left(\sigma / v_{i}\right)-1}+\left|x_{j}\right|^{\left(\sigma / v_{j}\right)-1}\right] .
\end{aligned}
$$

Hence, for $\forall 1 \leq k \neq j \leq i-1$, combining (A.19) with (A.20) yields

$$
\left|\frac{\partial^{2}\left[\xi_{i}\right]^{\sigma / v_{i}}}{\partial x_{k} \partial x_{j}}\right| \leq K_{i k l}\left(\bar{x}_{i}\right)\left[\sum_{l=1}^{i-1}\left|\zeta_{l}\right|+\left|x_{k}\right|^{\left(\sigma / v_{k}\right)-1}+\left|x_{j}\right|^{\left(\sigma / v_{j}\right)-1}\right] \text {. }
$$

Then, for $\forall 1 \leq k \neq j \leq i-1$, it can be deduced from (A.21) and Lemma 3 that

$$
\begin{aligned}
\frac{1}{2} \frac{\partial^{2} \Psi_{i}}{\partial x_{k} \partial x_{j}} g_{k}^{T} g_{j} \leq & \frac{4 \sigma-\mu-v_{i}}{\sigma} K_{i k j}\left(\bar{x}_{i}\right)\left[\sum_{l=1}^{i-1}\left|\varsigma_{l}\right|+\left|x_{k}\right|^{\left(\sigma / v_{k}\right)-1}+\left|x_{j}\right|^{\left(\sigma / v_{j}\right)-1}\right] \times\left|\varsigma_{i}\right|^{\left(3 \sigma-\mu-v_{i}\right) / \sigma}\left|x_{i}-\xi_{i}\right| \times \widetilde{\eta}_{k}\left(\sum_{m=1}^{k}\left|\varsigma_{m}\right|^{\left(2 v_{k}+\mu\right) / 2 \sigma}\right) \\
& \times \widetilde{\eta}_{j}\left(\sum_{m=1}^{j}\left|\varsigma_{m}\right|^{\left(2 v_{j}+\mu\right) / 2 \sigma}\right)+\frac{\left(4 \sigma-\mu-v_{i}\right)\left(3 \sigma-\mu-v_{i}\right)}{\sigma^{2}} \times\left[M_{i k}\left(\bar{x}_{i}\right) \sum_{l=1}^{i-1}\left|\varsigma_{l}\right|^{1-\left(v_{k} / \sigma\right)}\right] \times\left[M_{i j}\left(\bar{x}_{i}\right) \sum_{l=1}^{i-1}\left|\varsigma_{l}\right|^{1-\left(v_{j} / \sigma\right)}\right] \\
& \times\left|\varsigma_{i}\right|^{\left(2 \sigma-\mu-v_{i}\right) / \sigma}\left|x_{i}-\xi_{i}\right| \times \widetilde{\eta}_{k}\left(\sum_{m=1}^{k}\left|\varsigma_{m}\right|^{\left(2 v_{k}+\mu\right) / 2 \sigma}\right) \times \widetilde{\eta}_{j}\left(\sum_{m=1}^{j}\left|\varsigma_{m}\right|^{\left(2 v_{j}+\mu\right) / 2 \sigma}\right) \\
\leq & \frac{1}{32(i-1)(i-2)} \sum_{l=1}^{i-1} \varsigma_{l}^{4}+\widetilde{H}_{i 4 k j}\left(\bar{x}_{i}\right) \varsigma_{i}^{4},
\end{aligned}
$$

where $\widetilde{H}_{i 4 k j}(\cdot) \geq 0$ are $C^{2}$ functions. Hence, one has

$$
\frac{1}{2} \sum_{k, j=1, k \neq j}^{i-1} \frac{\partial^{2} \Psi_{i}}{\partial x_{k} \partial x_{j}} g_{k}^{T} g_{j} \leq \frac{1}{32} \sum_{l=1}^{i-1}\left|\zeta_{l}^{4}\right|+H_{i 42}\left(\bar{x}_{i}\right) \zeta_{l}^{4},
$$

where $H_{i 42}(\cdot)=\sum_{k, j=1, k \neq j}^{i-1} H_{i 4 k j}$. Similarly, applying (A.10), Proposition A.1 and Lemma 3, we get

$$
\begin{array}{r}
\sum_{j=1}^{i-1} \frac{\partial^{2} \Psi_{i}}{\partial x_{j} \partial x_{i}} g_{j}^{T} g_{i} \leq \frac{1}{32} \sum_{l=1}^{i-1}\left|\zeta_{l}^{4}\right|+H_{i 43}\left(\bar{x}_{i}\right) \zeta_{l}^{4}, \\
\frac{\partial^{2} \Psi_{i}}{\partial x_{i}^{2}}\left\|g_{i}\right\|^{2} \leq \frac{1}{32} \sum_{l=1}^{i-1}\left|\zeta_{l}^{4}\right|+H_{i 43}\left(\bar{x}_{i}\right) \zeta_{l}^{4},
\end{array}
$$

where $H_{i 43}(\cdot)$ and $H_{i 44}(\cdot)$ are nonnegative $C^{2}$ functions. Substituting (A.18), (A.23)-(A.25) into (A.15) directly infers (A.14). Till now, the proof of Proposition A.5 is finished.

Combining Propositions A.1-A.5 with (A.2) yields

$$
\begin{aligned}
\ell V_{i} \leq & -\frac{1}{2} G\left(x_{1}\right) \zeta_{1}^{4}-(n+1-i) \sum_{l=1}^{i-1} \zeta_{l}^{4}+H_{i}\left(\bar{x}_{i}\right) \zeta_{l}^{4} \\
& +\vartheta_{i}(t)\left[\zeta_{i}\right]^{\left(4 \sigma-\mu-v_{i}\right) / \sigma} \xi_{i+1}^{q_{i}}+\vartheta_{i}(t)\left[\zeta_{i}\right]^{\left(4 \sigma-\mu-v_{i}\right) / \sigma} \\
& \cdot\left(x_{i+1}^{q_{i}}-\xi_{i+1}^{q_{i}}\right),
\end{aligned}
$$

where $H_{i}(\cdot)=\sum_{\tau=1}^{4} H_{i \tau}(\cdot) \geq 0$ is a $C^{2}$ functions.

Design

$$
\begin{aligned}
\xi_{i+1} & =-\lambda_{i}\left(\bar{x}_{i}\right)\left[\varsigma_{i}\right\rceil^{\left(v_{i}+1\right) / \sigma} \text { with } \lambda_{i}\left(\bar{x}_{i}\right) \\
& =\left[\frac{n-i+1+H_{i}\left(\bar{x}_{i}\right)}{\underline{\vartheta}_{i}}\right]^{1 / q_{i}}>0 .
\end{aligned}
$$

Then, substituting the value of $\xi_{i+1}$ into (A.27) yields that (40) holds. 


\section{Data Availability}

No data were used to support this study.

\section{Conflicts of Interest}

The authors declare that they have no conflicts of interest.

\section{Acknowledgments}

This work was supported by the Project of Anhui Province Outstanding Young Talent Support Program with Grant no. gxyq2018089.

\section{References}

[1] S. C. Tong, X. Min, and Y. X. Li, "Observer-based adaptive fuzzy tracking control for strict-feedback nonlinear systems with unknown control gain functions," IEEE Transactions on Cybernetics, 2020.

[2] S. Tong and Y. Li, "Robust adaptive fuzzy backstepping output feedback tracking control for nonlinear system with dynamic uncertainties," Science China Information Sciences, vol. 53, no. 2, pp. 307-324, 2010.

[3] D. Yang, X. Li, and J. Qiu, "Output tracking control of delayed switched systems via state-dependent switching and dynamic output feedback," Nonlinear Analysis: Hybrid Systems, vol. 32, pp. 294-305, 2019.

[4] X. Li, X. Yang, and T. Huang, "Persistence of delayed cooperative models: impulsive control method," Applied Mathematics and Computation, vol. 342, pp. 130-146, 2019.

[5] H. Shen, F. Li, H. Yan, H. R. Karimi, and H.-K. Lam, "Finitetime event-triggered $\$ \backslash$ mathcal $\{\mathrm{H}\} \_\{\text {infty }\}$ \$ control for T-S fuzzy markov jump systems," IEEE Transactions on Fuzzy Systems, vol. 26, no. 5, pp. 3122-3135, 2018.

[6] H. Shen, Z. G. Huang, J. D. Cao, and J. H. Park, "Exponential filtering for continuous-time switched neural networks under persistent Dwell-Time switching regularity," IEEE Transactions on Cybernetics, vol. 50, 2020.

[7] S. Ding, A. Levant, and S. Li, "Simple homogeneous slidingmode controller," Automatica, vol. 67, no. 5, pp. 22-32, 2016.

[8] K. Q. Mei and S. H. Ding, "Second-order sliding mode controller design subject to an upper-triangular structure," IEEE Transactions on Systems, Man, and Cybernetics: Systems, 2019.

[9] X. Liu, X. Su, P. Shi, and C. Shen, "Observer-based sliding mode control for uncertain fuzzy systems via event-triggered strategy," IEEE Transactions on Fuzzy Systems, vol. 27, no. 11, pp. 2190-2201, 2019.

[10] S. Ding and S. Li, "Second-order sliding mode controller design subject to mismatched term," Automatica, vol. 77, pp. 388-392, 2017.

[11] Z. F. Li, T. S. Li, G. Feng, R. Zhao, and Q. H. Shan, "Neural network-based adaptive control for pure-feedback stochastic nonlinear systems with time-varying delays and dead-zone input," Systems, Man, and Cybernetics: Systems, 2018.

[12] T. S. Li, Z. F. Li, D. Wang, and C. L. P. Chen, "Outputfeedback adaptive neural control for stochastic nonlinear time-varying delays systems with unknown control directions," IEEE Transactions on Neural Networks and Learning Systems, vol. 26, no. 6, pp. 1188-1201, 2015.

[13] J. Yin and S. Khoo, "Continuous finite-time state feedback stabilizers for some nonlinear stochastic systems," International Journal of Robust and Nonlinear Control, vol. 25, no. 11, pp. 1581-1600, 2015.
[14] Y. Li, K. Li, and S. Tong, "Finite-time adaptive fuzzy output feedback dynamic surface control for MIMO nonstrict feedback systems," IEEE Transactions on Fuzzy Systems, vol. 27, no. 1, pp. 96-110, 2019.

[15] L. Fang, L. Ma, S. Ding, and D. Zhao, "Finite-time stabilization for a class of high-order stochastic nonlinear systems with an output constraint," Applied Mathematics and Computation, vol. 358, pp. 63-79, 2019.

[16] W. Li, X. Liu, and S. Zhang, "Further results on adaptive statefeedback stabilization for stochastic high-order nonlinear systems," Automatica, vol. 48, no. 8, pp. 1667-1675, 2012.

[17] W. T. Zha, J. Y. Zhai, and S. M. Fei, "Output feedback control for a class of stochastic high-order nonlinear systems with time-varying delays," International Journal of Robust and Nonlinear Control, vol. 24, no. 16, pp. 2243-2260, 2014.

[18] X. Zhao, X. Wang, G. Zong, and X. Zheng, “Adaptive neural tracking control for switched high-order stochastic nonlinear systems," IEEE Transactions on Cybernetics, vol. 47, no. 10, pp. 3088-3099, 2017.

[19] Z. Song and J. Zhai, "Decentralized output feedback stabilization for switched stochastic high-order nonlinear systems with time-varying state/input delays," ISA Transactions, vol. 90, pp. 64-73, 2019.

[20] L. Liu, S. Xu, X.-J. Xie, and B. Xiao, "Observer-based decentralized control of large-scale stochastic high-order feedforward systems with multi time delays," Journal of the Franklin Institute, vol. 356, no. 16, pp. 9627-9645, 2019.

[21] B. Niu, M. Liu, and A. Li, "Global adaptive stabilization of stochastic high-order switched nonlinear non-lower triangular systems," Systems \& Control Letters, vol. 136, p. 104596, 2020.

[22] J. H. Park, H. Shen, X. H. Chang, and T. H. Lee, Recent Advances in Control and Filtering of Dynamic Systems with Constrained Signals, Springer, Berlin, Germany, 2019.

[23] L. Fang, L. Ma, S. Ding, and D. Zhao, "Robust finite-time stabilization of a class of high-order stochastic nonlinear systems subject to output constraint and disturbances," International Journal of Robust and Nonlinear Control, vol. 29, no. 16, pp. 5550-5573, 2019.

[24] K. P. Tee, S. S. Ge, and E. H. Tay, "Barrier Lyapunov Functions for the control of output-constrained nonlinear systems," Automatica, vol. 45, no. 4, pp. 918-927, 2009.

[25] K. P. Tee, B. Ren, and S. S. Ge, "Control of nonlinear systems with time-varying output constraints," Automatica, vol. 47, no. 11, pp. 2511-2516, 2011.

[26] L. D. Fang, L. Ma, S. H. Ding, and J. H. Park, "Finite-time stabilization of high-order stochastic nonlinear systems with asymmetric output constraints," IEEE Transactions on Systems, Man, and Cybernetics: Systems, 2020.

[27] Y.-J. Liu and S. Tong, "Barrier Lyapunov Functions-based adaptive control for a class of nonlinear pure-feedback systems with full state constraints," Automatica, vol. 64, pp. 70-75, 2016.

[28] S. Ding, W.-H. Chen, K. Mei, and D. J. Murray-Smith, "Disturbance observer design for nonlinear systems represented by input-output models," IEEE Transactions on Industrial Electronics, vol. 67, no. 2, pp. 1222-1232, 2020.

[29] S. H. Ding, J. H. Park, and C. C. Chen, "Second-order sliding mode controller design with output constraint," Automatica, vol. 112, Article ID 108704, 2020.

[30] S. Ding, K. Mei, and S. Li, "A new second-order sliding mode and its application to nonlinear constrained systems," IEEE Transactions on Automatic Control, vol. 64, no. 6, pp. 25452552, 2019. 
[31] X. Yang, X. D. Li, X. Li, and Q. Xi, "Review of stability and stabilization for impulsive delayed systems," Mathematical Biosciences \& Engineering, vol. 15, no. 6, pp. 1495-1515, 2018.

[32] Q. K. Duan, S. H. Ding, and X. H. Yu, "Composite supertwisting sliding mode control design for PMSM speed regulation problem based on a novel disturbance observer," IEEE Transactions on Energy Conversion, 2020.

[33] L. Liu, W. X. Zheng, and S. H. Ding, "An adaptive SOSM controller design by using a sliding-mode-based filter and its application to buck converter," IEEE Transactions on Circuits and Systems I: Regular Papers, vol. 67, no. 7, pp. 2409-2418, 2020.

[34] R. Ma, Y. Liu, S. Zhao, and J. Fu, "Finite-time stabilization of a class of output-constrained nonlinear systems," Journal of the Franklin Institute, vol. 352, no. 12, pp. 5968-5984, 2015.

[35] C. C. Chen, "A unified approach to finite-time stabilization of high-order nonlinear systems with and without an output constraint," International Journal of Robust and Nonlinear Control, vol. 29, no. 2, pp. 393-407, 2019.

[36] C. C. Chen and G. S. Chen, "A new approach to stabilization of high-order nonlinear systems with an asymmetric output constraint," International Journal of Robust and Nonlinear Control, vol. 30, no. 2, pp. 756-775, 2020.

[37] C. C. Chen and Z. Y. Sun, "A unified approach to finite-time stabilization of high-order nonlinear systems with an asymmetric output constraint," Automatica, vol. 111, Article ID 108581, 2020.

[38] S. Huang and Z. Xiang, "Finite-time stabilisation of a class of switched nonlinear systems with state constraints," International Journal of Control, vol. 91, no. 6, pp. 1300-1313, 2018.

[39] X. Jin, "Adaptive fault tolerant tracking control for a class of stochastic nonlinear systems with output constraint and actuator faults," Systems \& Control Letters, vol. 107, pp. 100-109, 2017.

[40] Y.-J. Liu, S. Lu, S. Tong, X. Chen, and C. L. P. Chen, “Adaptive control-based barrier Lyapunov functions for a class of stochastic nonlinear systems with full state constraints," Automatica, vol. 87, pp. 83-93, 2018.

[41] J. Li, J. Xia, W. Sun, G. Zhuang, and Z. Wang, "Finite-time tracking control for stochastic nonlinear systems with full state constraints," Applied Mathematics and Computation, vol. 338, pp. 207-220, 2018.

[42] L. D. Fang, H. S. Ding, J. H. Park, and L. Ma, "Adaptive fuzzy control for nontriangular stochastic high-order nonlinear systems subject to asymmetric output constraints," IEEE Transactions on Cybernetics, 2020.

[43] Y. Liu, H. Ma, and H. Ma, "Adaptive fuzzy fault-tolerant control for uncertain nonlinear switched stochastic systems with time-varying output constraints," IEEE Transactions on Fuzzy Systems, vol. 26, no. 5, pp. 2487-2498, 2018.

[44] B. Niu, W. Ding, H. Li, and X. Xie, "A novel neural-networkbased adaptive control scheme for output-constrained stochastic switched nonlinear systems," IEEE Transactions on Systems, Man, and Cybernetics: Systems, vol. 49, no. 2, pp. 418-432, 2019.

[45] L. D. Fang, S. H. Ding, J. H. Park, and L. Ma, “Adaptive fuzzy control for stochastic high-order nonlinear systems with output constraints," IEEE Transactions on Fuzzy Systems, 2020.

[46] Y. Li and S. Tong, "Adaptive fuzzy output constrained control design for multi-input multioutput stochastic nonstrictfeedback nonlinear systems," IEEE Transactions on Cybernetics, vol. 47, no. 12, pp. 4086-4095, 2017. 